\title{
Structural Properties of Faces of the Cone of Copositive Matrices
}

\author{
Olga Kostyukova ${ }^{1,+}$ and Tatiana Tchemisova ${ }^{2, *,+}$ (D) \\ 1 Institute of Mathematics, National Academy of Sciences of Belarus, Surganov Str. 11, 220072 Minsk, Belarus; \\ kostyukova@im.bas-net.by \\ 2 Mathematical Department, Campus Universitario Santiago, University of Aveiro, 3810-193 Aveiro, Portugal \\ * Correspondence: tatiana@ua.pt \\ + These authors contributed equally to this work.
}

\section{check for}

updates

Citation: Kostyukova, O.; Tchemisova, T. Structural Properties of Faces of the Cone of Copositive Matrices. Mathematics 2021, 9, 2698. https://doi.org/10.3390/math9212698

Academic Editor: Néstor Thome

Received: 21 September 2021

Accepted: 19 October 2021

Published: 24 October 2021

Publisher's Note: MDPI stays neutral with regard to jurisdictional claims in published maps and institutional affiliations.

Copyright: (C) 2021 by the authors. Licensee MDPI, Basel, Switzerland. This article is an open access article distributed under the terms and conditions of the Creative Commons Attribution (CC BY) license (https:/ / creativecommons.org/licenses/by/ $4.0 /)$.

\begin{abstract}
In this paper, we study the properties of faces and exposed faces of the cone of copositive matrices (copositive cone), paying special attention to issues related to their geometric structure. Based on the concepts of zero and minimal zero vectors, we obtain several explicit representations of faces of the copositive cone and compare them. Given a face of the cone of copositive matrices, we describe the subspace generated by that face and the minimal exposed face containing it. Summarizing the results obtained in the paper, we systematically show what information can be extracted about the given copositive face in the case of incomplete data. Several examples for illustrating the main findings of the paper and also for justifying the usefulness of the developed approach to the study of the facial structure of the copositive cone are discussed.
\end{abstract}

Keywords: copositive matrices; completely positive matrices; copositive cone; minimal exposed cone

\section{Introduction}

The paper is devoted to the study of the facial structure of the cone $\mathcal{C O P}{ }^{p}$ of copositive matrices and is motivated by our main task for the future: the investigation of optimality conditions for the problems of copositive programming $(\mathrm{CoP})$ and the search for new dual formulations for them.

Copositive problems attract the attention of researchers because they have many interesting applications (see, for example, [1-3], and the references therein). Copositive problems belong to the class of conic optimization problems consisting of minimizing a convex function over the intersection of an affine subspace and a convex cone. Conic optimization leads to simple and ingenious formulations of many optimization problems, since it allows one to explicitly describe many important types of constraints in a way that is natural for many applied problems. CoP is sometimes seen as a generalization of Semidefinite Programming (SDP) and a special case of Semi-infinite Programming (SIP), whose important applications are well-known $[4,5]$. Note that the cone $\mathcal{C O P}^{p}$ can be considered as a generalization of the cone of semidefinite matrices, but is more complex and its facial structure is less studied than that of the SDP cone.

For convex conic optimization problems, the study of the facial structure of the corresponding cone is crucial, since the properties of its faces can be used for

(1) creation of regularization algorithms (facial reduction algorithms) and their justification,

(2) development and understanding of duality theory,

(3) obtaining optimality conditions,

which are important issues for any optimization problem. For example, in the papers [6-9], the facial structure of the cone of semidefinite matrices is used to solve the above problems. Currently, some interesting results have been obtained for special classes of faces of the cone $\mathcal{C O P}{ }^{p}$ in [10-12], but in general this problem has not yet been completely resolved. There are many open problems in studying the facial structure of this cone $[3,13]$. The structure of the faces of other cones is studied in [14]. 
In our previous work (see [15] and the references in it), we defined the zero and minimal zero vectors of a given convex subset of the cone $\mathcal{C O P}^{p}$. These definitions generalize the concepts of zeros and minimal zeros of copositive matrices, which were used in [11] to study the properties of the minimal and maximal faces of $\mathcal{C O P}{ }^{p}$. In [15] we showed how with the help of zeros and minimal zeros one can obtain different representations of the faces of the cone $\mathcal{C O P}^{p}$ and the corresponding dual cones. We also described the minimal face of $\mathcal{C O P}^{p}$ containing a given convex subset of this cone, and proved some propositions that can be useful for obtaining equivalent representations of feasible sets of copositive problems and creating new numerical methods based on the regularization approach.

In this paper, we will use some of our previous results to give a deeper understanding of the properties of faces of the cone of copositive matrices. In particular, for a given face of $\mathcal{C O P}^{p}$, we will describe the minimal exposed face containing this face, and examine the subspace generated by it. Some special classes of faces of the cone $\mathcal{C O P}{ }^{p}$ and their properties will also be considered. We will show how one can get as much useful information as possible about the properties of a face of $\mathcal{C O P}^{p}$ in case of incomplete data about this face.

The paper is structured as follows. Section 1 hosts an introduction. Section 2 contains the basic notations and some preliminary results for representing the faces of the cone of copositive matrices in terms of their zeros, which will be used in the following sections. Several examples illustrating these results are also presented in section 2 . In section 3 , we investigate the structural properties of the faces of the cone $\mathcal{C O P}^{p}$ and the corresponding subspaces. In particular, for a given face of $\mathcal{C O P}{ }^{p}$, we describe the subspace generated by that face and the minimal exposed face containing it. In section 4 , we discuss the faces of $\mathcal{C O P}^{p}$, which are defined by singleton sets. Section 5 contains several additional examples that justify the usefulness of the approach developed in the paper to the study of the facial structure of the cone $\mathcal{C O P}^{p}$. In section 6 , we present the main conclusions and systematically show what information about the face of the copositive cone can be extracted from some incomplete data about this face, based on the results obtained in the paper.

\section{Basic Notation and Preliminary Results}

\subsection{Cones and Faces: General Definitions}

Given a finite-dimensional space $\mathfrak{X}$, let us recall some general definitions.

A set $C \subset \mathfrak{X}$ is convex if for any $x, y \in C$ and any $\alpha \in[0,1]$, it holds

$$
\alpha x+(1-\alpha) y \in C
$$

Given a set $\mathcal{B} \subset \mathfrak{X}$, denoted by conv $\mathcal{B}$ its convex hull, i.e., the minimal (by inclusion) convex set, containing this set, and by $\operatorname{span}(\mathcal{B})$ its span, i.e., the smallest linear subspace containing $\mathcal{B}$. A set $K \subset \mathfrak{X}$ is a cone if for any $x \in K$ and any $\alpha>0$, it holds $\alpha x \in K$.

A nonempty convex subset $F$ of a convex closed set $C \subset \mathfrak{X}$ is called face of $C$ if from the condition $\alpha x+(1-\alpha) y \in F$ with $x, y \in C$ and $\alpha \in(0,1)$, it follows that $x, y \in F$. The standard notation $F \unlhd C$ is used to denote that $F$ is a face of the set $C$. We say that a face $F$, $F \unlhd C$, is proper if $F \neq C$ and write in this case $F \triangleleft C$.

A face $F$ of a closed convex set $C \subset \mathfrak{X}$ is called exposed if it can be represented as intersection of $C$ with a supporting hyperplane, i.e., there exist $y \in \mathfrak{X}$ and $d \in \mathbb{R}$ such that for all $x \in C$ it holds: $\langle y, x\rangle \geq d$ and $\langle y, x\rangle=d$ iff $x \in F$. Every exposed face should also be a face. Given a face $F$ of a set $C$, the minimal (by inclusion) exposed face containing $F$ will be called here the minimal exposed face for that face.

Given a cone $K \subset \mathfrak{X}$, its dual cone $K^{*}$ is given by

$$
K^{*}:=\{x \in \mathfrak{X}:\langle x, y\rangle \geq 0 \forall y \in K\} .
$$

Here, and in what follows, the notation := means that the item on the left-hand side is being defined to be what is on the right-hand side. Similarly, the notation =: means that the item on the right-hand side is being defined to be what is on the left-hand side. 


\subsection{The Cone of Copositive Matrices and Its Faces}

In this paper, we are dealing with special classes of cones of matrices, in particular, with the cones of copositive and completely positive matrices.

Given an integer $p>1$, consider the vector space $\mathbb{R}^{p}$ with the standard orthogonal basis $\left\{\mathbf{e}_{k}, k=1,2, \ldots, p\right\}$. This is denoted by $\mathbb{R}_{+}^{p} ;$ the set of all $p$-vectors with non-negative components is denoted by $\mathcal{S}^{p}$ - the space of real symmetric $p \times p$ matrices. The space $\mathcal{S}^{p}$ is considered here as a vector space with the trace inner product $A \bullet B:=\operatorname{trace}(A B)$.

Let $\mathcal{C O P}{ }^{p}$ denote the cone of symmetric copositive $p \times p$ matrices:

$$
\mathcal{C O P}{ }^{p}:=\left\{D \in \mathcal{S}^{p}: \mathbf{t}^{\top} D \mathbf{t} \geq 0 \forall \mathbf{t} \in \mathbb{R}_{+}^{p}\right\} .
$$

Consider a compact subset of $\mathbb{R}_{+}^{p}$ in the form of the simplex

$$
T:=\left\{\mathbf{t} \in \mathbb{R}_{+}^{p}: \mathbf{e}^{\top} \mathbf{t}=1\right\}
$$

with $\mathbf{e}=(1,1, \ldots, 1)^{\top} \in \mathbb{R}^{p}$. It is evident that the cone $\mathcal{C O P} \mathcal{P}^{p}$ can be equivalently described as follows:

$$
\mathcal{C O P} P^{p}=\left\{D \in \mathcal{S}^{p}: \mathbf{t}^{\top} D \mathbf{t} \geq 0 \forall \mathbf{t} \in T\right\} .
$$

The dual cone to $\mathcal{C O P}{ }^{p}$ is the cone of completely positive matrices, defined as

$$
\left.(\mathcal{C O P})^{p}\right)^{*} \mathcal{C P}^{p}:=\operatorname{conv}\left\{\mathbf{x x}^{\top}: \mathbf{x} \in \mathbb{R}_{+}^{p}\right\} .
$$

The cones of copositive and completely positive matrices are known to be proper cones, which means that they are closed, convex, pointed, and fully dimensional. The rest of this section is devoted to two alternative representations of the faces of these cones that will be used in this paper. Some of these results were obtained recently in [15]; others are new and have not been published before.

Given a vector $\mathbf{t}=\left(t_{k}, k \in[p]\right)^{\top} \in \mathbb{R}_{+}^{p}$ with $[p]:=\{1,2, \ldots, p\}$, consider support of $\mathbf{t}$, defined as

$$
\operatorname{supp}(\mathbf{t}):=\left\{k \in[p]: t_{k}>0\right\} .
$$

For a set $\mathcal{B} \subset \mathbb{R}^{p}$ and a point $\mathbf{l} \in \mathbb{R}^{p}$, the distance between them is denoted by $\rho(\mathbf{l}, \mathcal{B})$ : $\rho(\mathbf{1}, \mathcal{B}):=\min _{\boldsymbol{\tau} \in \mathcal{B}} \sum_{k \in P}\left|l_{k}-\tau_{k}\right|$

Consider a finite set of vectors in the above simplex $T$,

$$
V=\{\mathbf{t}(i), i \in I\} \subset T .
$$

This is denoted by

$$
\begin{gathered}
\sigma(V):=\min \left\{t_{k}(i), k \in \operatorname{supp}(\mathbf{t}(i)), i \in I\right\}>0, \\
\Omega(V):=\{\mathbf{t} \in T: \rho(\mathbf{t}, \operatorname{conv} V) \geq \sigma(V)\} .
\end{gathered}
$$

If the set $I$ in (2) is empty, we consider that $V=\varnothing$ and $\Omega(V)=T$.

The following results were obtained in [15].

Lemma 1 (Theorem 1 in [15]). For any $D \in \mathcal{S}^{p}$ and any set $V$ defined in (2), the inequalities

$$
D \mathfrak{t}(i) \geq 0 \quad \forall i \in I \text { and } \mathbf{t}^{\top} D \mathbf{t} \geq 0 \quad \forall \mathbf{t} \in \Omega(V),
$$

imply the inequalities

$$
\mathbf{t}^{\top} D \mathbf{t} \geq 0 \quad \forall \mathbf{t} \in T
$$


Lemma 2 (Proposition 2 in [15]). Any face $\mathcal{K}$ of the cone $\mathcal{C O P}^{p}$ can be presented as follows:

$$
\begin{aligned}
\mathcal{K} & =\mathcal{K}(V, \mathcal{L}):=\mathcal{C O} \mathcal{P}^{p} \cap \mathcal{H}(V, \mathcal{L}) \\
& =\left\{D \in \mathcal{C O} \mathcal{P}^{p}: \mathbf{e}_{k}^{\top} D \mathfrak{t}(i)=0, k \in L(i) ; \mathbf{e}_{k}^{\top} D \mathfrak{t}(i) \geq 0, k \in[p] \backslash L(i), i \in I\right\},
\end{aligned}
$$

where

$$
\mathcal{H}(V, \mathcal{L}):=\left\{D \in \mathcal{S}^{p}: \mathbf{e}_{k}^{\top} D \mathfrak{t}(i)=0, k \in L(i), i \in I\right\}
$$

with some vector set $V$ given in (2) and some set

$$
\mathcal{L}:=\{L(i), i \in I\},
$$

whose elements are the sets $L(i), i \in I$, satisfying the conditions $\operatorname{supp}(\mathbf{t}(i)) \subset L(i) \subset[p]$.

In what follows, we assume that any face of $\mathcal{C O P} \mathcal{P}^{p}$ is given in the form (5).

The pair of sets $\{V, \mathcal{L}\}$ will be called here the pair of defining sets for the face $\mathcal{K}(V, \mathcal{L})$. It is evident that if $I=\varnothing$, then $\mathcal{K}(V, \mathcal{L})=\mathcal{C O} \mathcal{P}^{p}$. If $\mathcal{K}(V, \mathcal{L}) \triangleleft \mathcal{C O} \mathcal{P}^{p}$, then $I \neq \varnothing$ and hence $V \neq \varnothing, \mathcal{L} \neq \varnothing$. This case is more interesting for research.

Remark 1. The subspace $\mathcal{H}(V, \mathcal{L})$ defined in (6) can be equivalently described as follows:

$$
\mathcal{H}(V, \mathcal{L})=\left\{D \in \mathcal{S}^{p}: D \bullet\left(\mathbf{e}_{k}(\mathbf{t}(i))^{\top}+\mathbf{t}(i) \mathbf{e}_{k}^{\top}\right)=0, k \in L(i), i \in I\right\} .
$$

Let $\mathcal{K} \unlhd \mathcal{C O P}^{p}$. According to the approach proposed in [15], we define the set of vectors satisfying the following conditions:

$$
T_{0}(\mathcal{K}):=\left\{\mathbf{t} \in T: \mathbf{t}^{\top} D \mathbf{t}=0 \forall D \in \mathcal{K}\right\} .
$$

The set $T_{0}(\mathcal{K})$ is empty if $\mathcal{K}=\mathcal{C O P}^{p}$, and is the union of a finite number of convex bounded polyhedra otherwise.

Consider the set

$$
V_{0}=V_{0}(\mathcal{K}):=\{\boldsymbol{\tau}(j), j \in J\},
$$

composed of all vertices of the set conv $T_{0}(\mathcal{K})$ where $J$ is the set of indices of vertices of the set conv $T_{0}(\mathcal{K})$.

In [15], the set $T_{0}(\mathcal{K})$ was called the set of zeros of the cone $\mathcal{K}$ and it was shown that this definition is equivalent to the concept of immobile indices of the constraints of certain related conic optimization problems (see, e.g., $[16,17])$. It was also shown that the set $V_{0}$ of vertices of conv $T_{0}(\mathcal{K})$ coincides with the set of minimal zeros of the matrix set $\mathcal{K}$. See [15] for the definition of minimal zeros of $\mathcal{K}$.

Given a cone $\mathcal{K} \triangleleft \mathcal{C O P}{ }^{p}$ and the corresponding set of minimal zeros (9), introduce the sets $M(j), j \in J$, and $\mathcal{M}$ :

$$
\begin{gathered}
M(j):=\left\{k \in[p]: \mathbf{e}_{k}^{\top} D \boldsymbol{\tau}(j)=0 \forall D \in \mathcal{K}\right\}, j \in J, \\
\mathcal{M}:=\{M(j), j \in J\} .
\end{gathered}
$$

The following lemma is based on the results of [15].

Lemma 3. Given a face $\mathcal{K} \triangleleft \mathcal{C O P}{ }^{p}$, let $T_{0}(\mathcal{K})$ be the set of zeros and $V_{0}=\{\boldsymbol{\tau}(j), j \in J\}$ the set of minimal zeros of $\mathcal{K}$. Let the sets $M(j), j \in J$, and $\mathcal{M}$ be defined in (10) and (11). Then $\mathcal{K}$ can be presented in the form

$$
\begin{aligned}
& \mathcal{K}=\mathcal{K}\left(V_{0}, \mathcal{M}\right):=\mathcal{C} \mathcal{O P}^{p} \cap \mathcal{H}\left(V_{0}, \mathcal{M}\right) \\
& =\left\{D \in \mathcal{C O P} \mathcal{P}^{p}: \mathbf{e}_{k}^{\top} D \boldsymbol{\tau}(j)=0, k \in M(j), \mathbf{e}_{k}^{\top} D \boldsymbol{\tau}(j) \geq 0, k \in[p] \backslash M(j), j \in J\right\},
\end{aligned}
$$


where the subspace $\mathcal{H}\left(V_{0}, \mathcal{M}\right)$ is defined by the following rules analogous to the rules (6):

$$
\mathcal{H}\left(V_{0}, \mathcal{M}\right):=\left\{D \in \mathcal{S}^{p}: \mathbf{e}_{k}^{\top} D \boldsymbol{\tau}(j)=0, k \in M(j), j \in J\right\},
$$

and there exists a matrix $\widehat{D} \in \mathcal{K}$ such that

$$
\mathbf{t}^{\top} \widehat{D} \mathbf{t}>0 \forall \mathbf{t} \in T \backslash T_{0}(\mathcal{K}), \quad \mathbf{e}_{k}^{\top} \widehat{D} \boldsymbol{\tau}(j)>0 \forall k \in[p] \backslash M(j), j \in J .
$$

Remark 2. From the above considerations, it follows that, in general, for a given $\mathcal{K} \unlhd \mathcal{C O P}{ }^{p}$, there may be several pairs $\{V, \mathcal{L}\}$ of defining sets (see Example 1 below). It is worth mentioning that, at the same time, there are faces of $\mathcal{C O P}{ }^{p}$, which have a unique pair of defining sets. We consider a set of such faces in Section 4.

To illustrate the concepts introduced above, consider the following example.

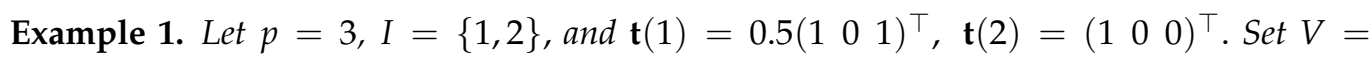
$\{\mathbf{t}(1), \mathbf{t}(2)\}, \mathcal{L}=\{L(1), L(2)\}$, where $L(1)=\{1,3\}, L(2)=\{1\}$, and consider the face $\mathcal{K}=\mathcal{K}(V, \mathcal{L})$ of $\mathcal{C O} \mathcal{P}^{3}$ defined in (5). It is simple to check this is correct:

$$
\mathcal{K}=\mathbf{K}:=\left\{D=\left(\begin{array}{lll}
0 & b & 0 \\
b & d & f \\
0 & f & 0
\end{array}\right), b \geq 0, d \geq 0, f \geq 0\right\},
$$

and $T_{0}(\mathcal{K}):=\left\{\mathbf{t} \in T: \mathbf{t}^{\top} D \mathbf{t}=0 \forall D \in \mathcal{K}\right\}=\left\{\mathbf{t}=\alpha \mathbf{e}_{1}+(1-\alpha) \mathbf{e}_{3}, \alpha \in[0,1]\right\}$. Hence, for this face $\mathcal{K}$, the set of minimal zeros (i.e., the set of all vertices of $\operatorname{conv} T_{0}(\mathcal{K})$ ) is as follows:

$$
V_{0}=\left\{\boldsymbol{\tau}(1)=\mathbf{e}_{1}, \boldsymbol{\tau}(2)=\mathbf{e}_{3}\right\}, J=\{1,2\},
$$

and the corresponding set $\mathcal{M}$ defined in (11) and (10) consists of two sets

$$
\mathcal{M}=\{M(1), M(2)\}, M(1)=M(2)=\{1,3\} .
$$

Note that, as it follows from Lemmas 2 and 3 , in this example we have $\mathcal{K}=\mathcal{K}(V, \mathcal{L})=\mathcal{K}\left(V_{0}, \mathcal{M}\right)$, but $V \neq V_{0}$ and $\mathcal{L} \neq \mathcal{M}$.

For the sets $V$ and $\mathcal{L}$ given in (2) and (7), consider the corresponding face $\mathcal{K}=\mathcal{K}(V, \mathcal{L})$ defined in (5). As above, let $V_{0}=\{\boldsymbol{\tau}(j), j \in J\}$ be the set of the minimal zeros of $\mathcal{K}$. Define the set

$$
\begin{gathered}
\mathcal{B}=\mathcal{B}\left(V, \mathcal{L}, V_{0}\right):= \\
\left\{D \in \mathcal{S}^{p}: \mathbf{e}_{k}^{\top} D \mathbf{t}(i)=0, k \in L(i), \mathbf{e}_{k}^{\top} D \mathbf{t}(i) \geq 0, k \in[p] \backslash L(i), i \in I\right. \\
\left.\mathbf{e}_{k}^{\top} D \boldsymbol{\tau}(j)=0, k \in \operatorname{supp}(\boldsymbol{\tau}(j)), \mathbf{e}_{k}^{\top} D \boldsymbol{\tau}(j) \geq 0, k \in[p] \backslash \operatorname{supp}(\boldsymbol{\tau}(j)), j \in J\right\} .
\end{gathered}
$$

Proposition 1. Given a face $\mathcal{K}=\mathcal{K}(V, \mathcal{L}) \triangleleft \mathcal{C O} \mathcal{P}^{p}$ with the corresponding set $V_{0}$ of minimal zeros, the following equalities hold true:

$$
\bar{M}(j):=\left\{k \in[p]: \mathbf{e}_{k}^{\top} D \boldsymbol{\tau}(j)=0 \forall D \in \mathcal{B}\right\}=M(j), j \in J,
$$

where the sets $M(j), j \in J$, are defined in (10), and the set $\mathcal{B}$ is defined in (16).

Proof. By construction, $\mathcal{K} \subset \mathcal{B}$. Then $\bar{M}(j) \subset M(j), j \in J$.

Let us show that $M(j) \subset \bar{M}(j), j \in J$. Suppose the contrary: there exists $j_{0} \in J$ such that $M\left(j_{0}\right) \not \subset \bar{M}\left(j_{0}\right)$. Consequently, there exists $k_{0} \in[p]$ such that $k_{0} \in M\left(j_{0}\right), k_{0} \notin \bar{M}\left(j_{0}\right)$. Then for some $\bar{D} \in \mathcal{B}$, it holds:

$$
\mathbf{e}_{k_{0}}^{\top} \bar{D} \boldsymbol{\tau}\left(j_{0}\right)>0, k_{0} \in[p] \backslash \operatorname{supp}\left(\boldsymbol{\tau}\left(j_{0}\right)\right) .
$$


By construction, we have $T_{0}(\mathcal{K}) \subset \operatorname{conv} T_{0}(\mathcal{K})=\operatorname{conv} V_{0}$ and by the definition of the set $\Omega(V)$ (see (4)), we have $\Omega\left(V_{0}\right)=\left\{\mathbf{t} \in T: \rho\left(\mathbf{t}\right.\right.$, conv $\left.\left.V_{0}\right) \geq \sigma\left(V_{0}\right)\right\}$ with $\sigma\left(V_{0}\right)>0$ defined in (3). This implies that

$$
\Omega\left(V_{0}\right) \cap T_{0}(\mathcal{K})=\varnothing .
$$

According to Lemma 3, there exists a matrix $\widehat{D} \in \mathcal{K}$ such that inequalities (14) hold true. Let us show that

$$
\mathbf{t}^{\top} \widehat{D} \mathbf{t}>0 \forall \mathbf{t} \in \Omega\left(V_{0}\right) .
$$

Indeed, suppose the contrary: there exists $\overline{\mathbf{t}} \in \Omega\left(V_{0}\right)$ such that $\overline{\mathbf{t}}^{\top} \hat{D} \overline{\mathbf{t}}=0$. Then it follows from (14) that $\overline{\mathbf{t}} \in T_{0}(\mathcal{K})$ and therefore $\overline{\mathbf{t}} \in T_{0}(\mathcal{K}) \cap \Omega\left(V_{0}\right)$. However, this contradicts (18). Thus, the inequalities (19) are proved.

For the above matrices $\widehat{D}$ and $\bar{D}$ denote $D(\alpha):=\widehat{D}+\alpha \bar{D}$, where $\alpha \in \mathbb{R}$. From (19) it follows that there exists $\alpha>0$ such that

$$
\mathbf{t}^{\top} D(\alpha) \mathbf{t} \geq 0 \quad \forall \mathbf{t} \in \Omega\left(V_{0}\right) \text { and } D(\alpha) \boldsymbol{\tau}(j) \geq 0, j \in J .
$$

According to Lemma 1, from the relations above, it follows $D(\alpha) \in \mathcal{C O} \mathcal{P}^{p}$. Hence, taking into account the fact that, by construction, it holds

$$
\mathbf{e}_{k}^{\top} D(\alpha) \mathbf{t}(i)=0, k \in L(i) ; \quad \mathbf{e}_{k}^{\top} D(\alpha) \mathbf{t}(i) \geq 0, k \in[p] \backslash L(i), i \in I,
$$

we conclude that $D(\alpha) \in \mathcal{K}$. It is simple to observe that

$$
\mathbf{e}_{k_{0}}^{\top} D(\alpha) \boldsymbol{\tau}\left(j_{0}\right)=\mathbf{e}_{k_{0}}^{\top} \widehat{D} \boldsymbol{\tau}\left(j_{0}\right)+\alpha \mathbf{e}_{k_{0}}^{\top} \bar{D} \boldsymbol{\tau}\left(j_{0}\right)>0 .
$$

However, on the other hand, from the relations $D(\alpha) \in \mathcal{K}, j_{0} \in J, k_{0} \in M\left(j_{0}\right)$, it follows that $\mathbf{e}_{k_{0}}^{\top} D(\alpha) \boldsymbol{\tau}\left(j_{0}\right)=0$. The resulting contradiction proves the proposition.

Let us compare the equivalent representations (10) and (17) of the sets $M(j), j \in J$. Note that the set $\mathcal{B}$ is polyhedral, but the set $\mathcal{K}$ is not (in general). Therefore, to construct the set $\bar{M}(j)$ for any $j \in J$, it is enough to solve several Linear Programming (LP) problems. Hence the representation (17) is more constructive than (10) and in the following we assume that the sets $M(j), j \in J$, are defined by rules (17).

\subsection{Exposed Faces of $\mathcal{C O P}{ }^{p}$}

At the beginning of this section (see Section 2.1), we presented the definition of exposed faces of a cone. Applying the results from [18] (see page 51), we can say that a face $\mathcal{K}$ is an exposed face of $\mathcal{C O P} \mathcal{P}^{p}$ if and only if there exists a matrix $A \in \mathcal{C P}{ }^{p}$ such that

$$
\mathcal{K}=\left\{D \in \mathcal{C O P}^{p}: D \bullet A=0\right\}
$$

The following proposition provides additional information about the structure of exposed faces of $\mathcal{C O P}{ }^{p}$.

Proposition 2. A face $\mathcal{K} \unlhd \mathcal{C O P}{ }^{p}$ is exposed iff it admits representation (5) with some sets $V$ given in (2) and $\mathcal{L}$ defined as follows:

$$
\mathcal{L}=\{L(i)=\operatorname{supp}(\mathbf{t}(i)), i \in I\} .
$$

Proof. As mentioned above, any exposed face $\mathcal{K} \unlhd \mathcal{C O P}^{p}$ can be presented in the form (20) with some $A \in \mathcal{C P}^{p}$. Being completely positive, the matrix $A$ admits the following representation:

$$
A=\sum_{i \in I} \gamma_{i} \mathbf{t}(i)(\mathbf{t}(i))^{\top}, \text { where } \gamma_{i}>0, \mathbf{t}(i) \in T, i \in I,|I| \leq p(p+1) / 2
$$


For any $D \in \mathcal{C O P}^{p}$ and any $A$ in the form (22), let us show the equivalence

$$
D \bullet A=0 \Longleftrightarrow \mathbf{e}_{k}^{\top} D \mathbf{t}(i)=0, k \in \operatorname{supp}(\mathbf{t}(i)), i \in I .
$$

It is evident that the conditions $D \in \mathcal{C} \mathcal{O P}^{p}$ and $D \bullet A=0$ imply $(\mathbf{t}(i))^{\top} D \mathfrak{t}(i)=0$, $i \in I$. Hence the vectors $\mathbf{t}(i), i \in I$, solve the optimization problem $\min _{\mathbf{t} \in T} \mathbf{t}^{\top} D \mathbf{t}$.

From the optimality conditions for the vectors $\mathbf{t}(i), i \in I$, in the problem above, it follows that $\mathbf{e}_{k}^{\top} D \mathbf{t}(i)=0, k \in \operatorname{supp}(\mathbf{t}(i)), i \in I$. Thus, we showed the implication

$$
D \bullet A=0 \Longrightarrow \mathbf{e}_{k}^{\top} D \mathfrak{t}(i)=0, k \in \operatorname{supp}(\mathfrak{t}(i)), i \in I,
$$

for any $D \in \mathcal{C O P}^{p}$ and any $A$ in the form (22).

On the other hand, it is obvious that the conditions $\mathbf{e}_{k}^{\top} D \mathbf{t}(i)=0, k \in \operatorname{supp}(\mathbf{t}(i)), i \in I$, imply $(\mathbf{t}(i))^{\top} D \mathfrak{t}(i)=0, i \in I$, and then $D \bullet A=\sum_{i \in I} \gamma_{i}(\mathbf{t}(i))^{\top} D \mathfrak{t}(i)=0$. Hence, we have shown that

$$
\mathbf{e}_{k}^{\top} D \mathbf{t}(i)=0, k \in \operatorname{supp}(\mathbf{t}(i)), i \in I \Longrightarrow D \bullet A=0 .
$$

For $D \in \mathcal{C O} \mathcal{P}^{p}$ and $A$ in the form (22), the equivalence of the conditions in (23) follows from the above-proven implications.

It is easy to see that (23) implies the equivalence of representations (20) and (5) with the sets $V$ and $\mathcal{L}$ defined in (2) and (21), and the proposition is proved.

The next lemma presents one known result that will be constructively proved here by representation (5).

Lemma 4. For any $\mathcal{K} \unlhd \mathcal{C O P}{ }^{p}$, there exists an exposed face $\mathcal{K}_{\text {exp }}$ of $\mathcal{C O P}{ }^{p}$ such that $\mathcal{K}$ is an exposed face of $\mathcal{K}_{\exp }$.

Proof. According to Lemma 2, any face $\mathcal{K}$ of the cone $\mathcal{C O P}^{p}$ admits the representation $\mathcal{K}=\mathcal{K}(V, \mathcal{L})($ see (5)), where $V$ and $\mathcal{L}$ are some sets defined in (2) and (7). Using these sets, let us form matrices

$$
A:=\sum_{i \in I} \mathbf{t}(i)(\mathbf{t}(i))^{\top} \text { and } B:=\sum_{i \in I} \sum_{k \in L(i)}\left(\mathbf{e}_{k}(\mathbf{t}(i))^{\top}+\mathbf{t}(i)\left(\mathbf{e}_{k}\right)^{\top}\right) .
$$

It is evident that $A \in \mathcal{C P}^{p}=\left(\mathcal{C O P}^{p}\right)^{*}$ and $B \in \mathcal{C O} \mathcal{P}^{p}$.

Consider the cone $\mathcal{K}_{\text {exp }}:=\left\{D \in \mathcal{C O} \mathcal{P}^{p}: D \bullet A=0\right\}$.

By construction, $\mathcal{K}_{\text {exp }}$ is an exposed face of $\mathcal{C O P}{ }^{p}$ and it easy to see that for any matrix $D \in \mathcal{K}_{\text {exp }}$, it holds

$$
\mathbf{e}_{k}^{\top} D \mathfrak{t}(i)=0, k \in \operatorname{supp}(\mathbf{t}(i)), \mathbf{e}_{k}^{\top} D \mathfrak{t}(i) \geq 0, k \in[p] \backslash \operatorname{supp}(\mathbf{t}(i)), i \in I .
$$

These relations imply that

$$
B \bullet D=2 \sum_{i \in I} \sum_{k \in L(i)} \mathbf{e}_{k}^{\top} D \mathfrak{t}(i) \geq 0 \forall D \in \mathcal{K}_{\exp } .
$$

Hence, we have shown that $B \in\left(\mathcal{K}_{\text {exp }}\right)^{*}$.

Now, consider the cone $\mathcal{K}_{*}:=\left\{D \in \mathcal{K}_{\exp }: D \bullet B=0\right\}$. By construction, $\mathcal{K}_{*}$ is an exposed face of the cone $\mathcal{K}_{\text {exp }}$ (which, in turn, is an exposed face of $\mathcal{C O P}{ }^{p}$ ) and $\mathcal{K}_{*}=\mathcal{K}$. The lemma is proved.

From the proof of Lemma 4 it follows that the face representation (5) allows one to explicitly describe an exposed face $\mathcal{K}_{\exp }$ mentioned in the lemma's formulation. 


\section{On the Structural Properties of the Faces of the Cone $\mathcal{C O P}{ }^{p}$}

\subsection{Subspaces Generated by Faces of $\mathcal{C O P}^{p}$}

Consider the face $\mathcal{K} \unlhd \mathcal{C O P} \mathcal{P}^{p}$ defined in (5) in the form $\mathcal{K}=\mathcal{K}(V, \mathcal{L})=\mathcal{C O} \mathcal{P}^{p} \cap$ $\mathcal{H}(V, \mathcal{L})$, where the subspace $\mathcal{H}(V, \mathcal{L})$ and the sets $V, \mathcal{L}$ are given in (6), (2), (7), respectively.

In the previous section, it was shown that the face $\mathcal{K}$ admits the alternative representation (12) via the subspace $\mathcal{H}\left(V_{0}, \mathcal{M}\right)$ described in (13) using the set $V_{0}$ of the minimal zeros of $\mathcal{K}$ and the set $\mathcal{M}$ defined in (11), (17). The following statements establish the relationship between the subspaces $\mathcal{H}(V, \mathcal{L}), \mathcal{H}\left(V_{0}, \mathcal{M}\right)$, and $\operatorname{span}(\mathcal{K})$.

Lemma 5. The inclusion $\mathcal{H}\left(V_{0}, \mathcal{M}\right) \subset \mathcal{H}(V, \mathcal{L})$ holds true.

Proof. For a fixed $i_{0} \in I$, consider $\mathbf{t}\left(i_{0}\right) \in V$. Since $\mathbf{t}\left(i_{0}\right) \in T_{0}(\mathcal{K}(V, \mathcal{L})) \subset \operatorname{conv} V_{0}$, we have

$$
\mathbf{t}\left(i_{0}\right)=\sum_{j \in J_{*}\left(i_{0}\right)} \alpha_{j} \tau(j) \text { with } \alpha_{j}>0, j \in J_{*}\left(i_{0}\right),
$$

for some $J_{*}\left(i_{0}\right) \subset J$. Then

$$
k \in L\left(i_{0}\right) \Longrightarrow k \in M(j) \forall j \in J_{*}\left(i_{0}\right) .
$$

For any $D \in \mathcal{H}\left(V_{0}, \mathcal{M}\right)$, taking into account (24) and (25), calculate

$$
\mathbf{e}_{k}^{\top} D \mathbf{t}\left(i_{0}\right)=\mathbf{e}_{k}^{\top} D \sum_{j \in J_{*}\left(i_{0}\right)} \alpha_{j} \boldsymbol{\tau}(j)=0 \forall k \in L\left(i_{0}\right) .
$$

Then $D \in \mathcal{H}(V, \mathcal{L})$ and consequently, $\mathcal{H}\left(V_{0}, \mathcal{M}\right) \subset \mathcal{H}(V, \mathcal{L})$. The lemma is proved.

The following example illustrates that, in general, $\mathcal{H}\left(V_{0}, \mathcal{M}\right) \neq \mathcal{H}(V, \mathcal{L})$.

Example 2. For $p=3$, denote $\mathbf{t}(1)=\left(\begin{array}{lll}1 / 3 & 1 / 3 & 1 / 3\end{array}\right)^{\top}, \mathbf{t}(2)=\left(\begin{array}{lll}0 & 1 & 0\end{array}\right)^{\top}, I=\left\{\begin{array}{ll}1,2 \\ V\end{array}\right.$, $V=\{\mathbf{t}(i), i \in I\}, \mathcal{L}=\{L(i), i \in I\}, L(1)=\{1,2,3\}, L(2)=\{2\}$. Then

$$
\begin{gathered}
\mathcal{H}(V, \mathcal{L})=\left\{\left(\begin{array}{ccc}
a & b & -a \\
b & 0 & -b \\
-a & -b & a
\end{array}\right), a \in \mathbb{R}, b \in \mathbb{R}\right\}, \\
\mathcal{K}(V, \mathcal{L})=\mathcal{C} \mathcal{O P}^{p} \cap \mathcal{H}(V, \mathcal{L})=\left\{\left(\begin{array}{ccc}
a & 0 & -a \\
0 & 0 & 0 \\
-a & 0 & a
\end{array}\right), a \geq 0\right\} .
\end{gathered}
$$

The face $\mathcal{K}(V, \mathcal{L})$ is exposed, since $L(i)=\operatorname{supp}(\mathbf{t}(i)), i=1,2$.

It is easy to check that $T_{0}(\mathcal{K}(V, \mathcal{L}))=\left\{\mathbf{t} \in T: t_{1}=t_{3}\right\}, V_{0}=\{\boldsymbol{\tau}(j), j \in J\}$, $\boldsymbol{\tau}(1)=\left(\begin{array}{lll}1 / 2 & 0 & 1 / 2\end{array}\right)^{\top}, \boldsymbol{\tau}(2)=\left(\begin{array}{lll}0 & 1 & 0\end{array}\right)^{\top}, J=\{1,2\}$, and $\mathcal{M}=\{M(j), j \in J\}$ with $M(j)=$ $\{1,2,3\}, j \in J$. Hence

$$
\mathcal{H}\left(V_{0}, \mathcal{M}\right)=\left\{\left(\begin{array}{ccc}
a & 0 & -a \\
0 & 0 & 0 \\
-a & 0 & a
\end{array}\right), a \in \mathbb{R}\right\} .
$$

Thus, we have $\mathcal{H}\left(V_{0}, \mathcal{M}\right) \subset \mathcal{H}(V, \mathcal{L}), \operatorname{dim} \mathcal{H}\left(V_{0}, \mathcal{M}\right)=1<\operatorname{dim} \mathcal{H}(V, \mathcal{L})=2$.

Theorem 1. Given $\mathcal{K} \unlhd \mathcal{C O} \mathcal{P}^{p}$, let $V_{0}=\{\boldsymbol{\tau}(j), j \in J\}$ be the set of minimal zeros of $\mathcal{K}$ and $\mathcal{M}$ the corresponding set defined in (11), (17). Then

$$
\operatorname{span}(\mathcal{K})=\mathcal{H}\left(V_{0}, \mathcal{M}\right)
$$

Proof. By definition, $\operatorname{span}(\mathcal{K}):=\left\{D=\sum_{j=1}^{p_{*}} \alpha_{j} D_{j}, \alpha_{j} \in \mathbb{R}, D_{j} \in \mathcal{K}, j=1, \ldots, p_{*}\right\}$, where $p_{*}=p(p+1) / 2$. From Lemma 3, it follows that $\operatorname{span}(\mathcal{K}) \subset \mathcal{H}\left(V_{0}, \mathcal{M}\right)$. 
Let us show that $\mathcal{H}\left(V_{0}, \mathcal{M}\right) \subset \operatorname{span}(\mathcal{K})$. Consider any $\bar{D} \in \mathcal{H}\left(V_{0}, \mathcal{M}\right)$. It follows from Lemma 3 that there exists $\widehat{D} \in \mathcal{K}$ satisfying relations (14). Consider a matrix $D(\lambda):=$ $\bar{D}+\lambda \widehat{D}$ for a sufficiently large $\lambda>0$. Then for $D(\lambda)$, it holds

$$
\mathbf{t}^{\top} D(\lambda) \mathbf{t} \geq 0 \forall \mathbf{t} \in \Omega\left(V_{0}\right), \quad D(\lambda) \boldsymbol{\tau}(j) \geq 0 \forall j \in J .
$$

It follows from the relations above and Lemma 1 that $D(\lambda) \in \mathcal{K}$. Hence

$$
\bar{D}=D(\lambda)-\lambda \widehat{D}, \text { where } D(\lambda) \in \mathcal{K}, \widehat{D} \in \mathcal{K} .
$$

This implies that $\bar{D} \in \operatorname{span}(\mathcal{K})$. The theorem is proved.

Corollary 1. In the notations of Theorem 1 , we can state that $\operatorname{dim} \mathcal{K}=\operatorname{dim} \mathcal{H}\left(V_{0}, \mathcal{M}\right)$.

To illustrate this corollary, let us again consider Example 2. It easy to see that in this example we have $\operatorname{dim} \mathcal{K}(V, \mathcal{L})=\operatorname{dim} \mathcal{H}\left(V_{0}, \mathcal{M}\right)=1$.

For a set $V$ in the form (2), construct the matrix $A=\sum_{i \in I} \mathbf{t}(i)(\mathbf{t}(i))^{\top}$ and consider the exposed face $\mathcal{K}:=\left\{D \in \mathcal{C O} \mathcal{P}^{p}: D \bullet A=0\right\}$. This face admits the following representations:

$$
\mathcal{K}=\mathcal{C O} \mathcal{P}^{p} \cap \mathcal{H}_{0}=\mathcal{C O} \mathcal{P}^{p} \cap \mathcal{H}_{*}=\mathcal{C O} \mathcal{P}^{p} \cap \mathcal{H}(V, \mathcal{L})=\mathcal{C O} \mathcal{P}^{p} \cap \mathcal{H}\left(V_{0}, \mathcal{M}\right),
$$

where $\mathcal{H}_{0}:=\left\{D \in \mathcal{S}^{p}: D \bullet A=0\right\}, \mathcal{H}_{*}:=\left\{D \in \mathcal{S}^{p}:(\mathbf{t}(i))^{\top} D \mathfrak{t}(i)=0, i \in I\right\}$, the set $\mathcal{L}$ is defined in (21), the set $V_{0}$ is the set of minimal zeros of $\mathcal{K}$, and the set $\mathcal{M}$ is given by (11) and (17).

For the subspaces $\mathcal{H}\left(V_{0}, \mathcal{M}\right), \mathcal{H}(V, \mathcal{L}), \mathcal{H}_{*}$, and $\mathcal{H}_{0}$, the inclusions

$$
\mathcal{H}\left(V_{0}, \mathcal{M}\right) \subset \mathcal{H}(V, \mathcal{L}) \subset \mathcal{H}_{*} \subset \mathcal{H}_{0}
$$

hold true. Hence, the representation $\mathcal{K}=\mathcal{C O} \mathcal{P}^{p} \cap \mathcal{H}\left(V_{0}, \mathcal{M}\right)$ is the best (in terms of the dimension of the subspace used for the representation).

\subsection{On the Construction of the Minimal Exposed Face Containing a Given Face}

In this subsection, given a face $\mathcal{K}$ of the cone $\mathcal{C O P}^{p}$, we will consider its minimal exposed face and show how it can be constructed using only the information about the corresponding sets $V_{0}$ and $\mathcal{M}$. After that, we will describe the minimal exposed face containing a given convex subset of $\mathcal{C O} \mathcal{P}^{p}$.

Suppose that for some face $\mathcal{K} \unlhd \mathcal{C O P}{ }^{p}$ (possibly unknown), the corresponding set $V_{0}$ of minimal zeros in the form (9), and the set $\mathcal{M}$ defined in (11), (17) are known. Thus, we consider that the finite data sets

$$
V_{0}=\{\boldsymbol{\tau}(j), j \in J\}, \mathcal{M}=\{M(j), j \in J\}
$$

are given.

First of all, let us note that using the known sets (28), one can explicitly present the face $\mathcal{K}=\mathcal{K}\left(V_{0}, \mathcal{M}\right)$ by the rule (12).

Having data (28), let us partition the index set $J$ into subsets $J(s)$ where $s \in S,|S| \geq 1$, such that

(a) $J=\bigcup_{s \in S} J(s)$,

(b) for any $s \in S$, it holds $\bigcup_{j \in J(s)} \operatorname{supp}(\boldsymbol{\tau}(j)) \subset M(i) \forall i \in J(s)$,

(c) if $|S| \geq 2$, then $\forall s \in S, \forall \bar{s} \in S, s \neq \bar{s}$, we have

$$
J(s) \backslash J(\bar{s}) \neq \varnothing, J(\bar{s}) \backslash J(s) \neq \varnothing,
$$


and $\forall i_{0} \in J(s) \backslash J(\bar{s}), \forall j_{0} \in J(\bar{s}) \backslash J(s) \quad \exists k_{0} \in \operatorname{supp}\left(\tau\left(i_{0}\right)\right), k_{0} \notin M\left(j_{0}\right)$.

Note that the conditions (b) and (c) are equivalent to the following conditions, respectively:

(b1) $(\boldsymbol{\tau}(j))^{\top} D \boldsymbol{\tau}(i)=0 \quad \forall j \in J(s), \forall i \in J(s), \forall D \in \mathcal{K}, \forall s \in S$;

(c1) if $|S| \geq 2$, then for all $\bar{s} \in S$ and all $s \in S, \bar{s} \neq s$,

the sets $J(s) \backslash J(\bar{s})$ and $J(\bar{s}) \backslash J(s)$ are non-empty and

$$
\exists \bar{D} \in \mathcal{K}:(\boldsymbol{\tau}(i))^{\top} \bar{D} \boldsymbol{\tau}(j)>0 \forall i \in J(s) \backslash J(\bar{s}), \forall j \in J(\bar{s}) \backslash J(s) .
$$

Proposition 3. Suppose that for a face $\mathcal{K} \unlhd \mathcal{C O P}{ }^{p}$, the sets (28) are known. Let $\{J(s), s \in S\}$ be the partition of the set $J$ such that the conditions $(\mathbf{a})-(\mathbf{c})$ are satisfied. Then the set $T_{0}(\mathcal{K})$ can be presented in the form

$$
T_{0}(\mathcal{K})=\bigcup_{s \in S} T_{0}(s, \mathcal{K})
$$

where

$$
T_{0}(s, \mathcal{K}):=\operatorname{conv}\{\boldsymbol{\tau}(j), j \in J(s)\}, \forall s \in S .
$$

Proof. For $s \in S$, consider $\mathbf{t} \in T_{0}(s, \mathcal{K})$. By construction, the vector $\mathbf{t}$ admits a representation

$$
\mathbf{t}=\sum_{j \in J(s)} \alpha_{j} \boldsymbol{\tau}(j), \alpha_{j} \geq 0, j \in J(s), \sum_{j \in J(s)} \alpha_{j}=1 .
$$

For any $D \in \mathcal{K}$, taking into account condition b1), let us calculate

$$
\mathbf{t}^{\top} D \mathbf{t}=\sum_{i \in J(s)} \sum_{j \in J(s)} \alpha_{j} \alpha_{i}(\boldsymbol{\tau}(j))^{\top} D \boldsymbol{\tau}(i)=0 .
$$

Then by definition, $\mathbf{t} \in T_{0}(\mathcal{K})$ and we have shown the inclusion

$$
\bigcup_{s \in S} T_{0}(s, \mathcal{K}) \subset T_{0}(\mathcal{K}) .
$$

Now let us show that $T_{0}(\mathcal{K}) \subset \bigcup_{s \in S} T_{0}(s, \mathcal{K})$. Consider $\mathbf{t} \in T_{0}(\mathcal{K})$. By construction,

$$
\mathbf{t}=\sum_{j \in J_{*}} \alpha_{j} \boldsymbol{\tau}(j), \quad \alpha_{j}>0, j \in J_{*} \subset J, \sum_{j \in J_{*}} \alpha_{j}=1 .
$$

If there exists $s_{0} \in S$ such that $J_{*} \subset J\left(s_{0}\right)$, then $\mathbf{t} \in T_{0}\left(s_{0}, \mathcal{K}\right)$ and, consequently, $\mathbf{t} \in \bigcup_{s \in S} T_{0}(s, \mathcal{K})$. Suppose that on the contrary, it holds

$$
J_{*} \not \subset J(s) \forall s \in S .
$$

Denoted by $J_{*}(s):=J_{*} \cap J(s), s \in S, S_{*}:=\left\{s \in S: J_{*}(s) \neq \varnothing\right\}$. It is evident that $\left|S_{*}\right| \geq 2$ and $J_{*}=\bigcup_{s \in S_{*}} J_{*}(s)$.

Let $\bar{s} \in S_{*}$ be such that

$$
\left|J_{*}(\bar{s})\right|=\max _{s \in S_{*}}\left|J_{*}(s)\right| .
$$

Denoted by $S_{*}(\bar{s}):=\left\{s \in S_{*}: J_{*}(s) \backslash J_{*}(\bar{s}) \neq \varnothing\right\}$.

If $S_{*}(\bar{s})=\varnothing$, then $J_{*}(s) \backslash J_{*}(\bar{s})=\varnothing \forall s \in S_{*} \Longrightarrow J_{*}(s) \subset J_{*}(\bar{s}) \forall s \in S_{*}$, and hence, $J_{*}=J_{*}(\bar{s}) \subset J(\bar{s})$. However, this contradicts (33). Therefore, $S_{*}(\bar{s}) \neq \varnothing$. 
Consider any $\hat{s} \in S_{*}(\bar{s})$. Suppose that $J_{*}(\bar{s}) \backslash J_{*}(\hat{s})=\varnothing$. Then $J_{*}(\bar{s}) \subset J_{*}(\hat{s})$, wherefrom, taking into account that by construction $J_{*}(\bar{s}) \neq J_{*}(\hat{s})$, we get the inequality $\left|J_{*}(\bar{s})\right|<$ $\left|J_{*}(\hat{s})\right|$ contradicting (34). Hence, we have shown that there exist $\bar{s} \in S_{*}$ and $\hat{s} \in S_{*}$ such that

$$
J_{*}(\bar{s}) \backslash J_{*}(\hat{s}) \neq \varnothing, \quad J_{*}(\hat{s}) \backslash J_{*}(\bar{s}) \neq \varnothing .
$$

Let $i_{0} \in J_{*}(\bar{s}) \backslash J_{*}(\hat{s})$. The condition $i_{0} \in J_{*}(\bar{s})$ implies the conditions $i_{0} \in J(\bar{s})$ and $i_{0} \in J_{*}$. The condition $i_{0} \notin J_{*}(\hat{s})$ is equivalent to the following one: $i_{0} \notin J(\hat{s}) \cap J_{*}$ wherefrom, taking into account that $i_{0} \in J_{*}$, we obtain $i_{0} \notin J(\hat{s})$. Thus, we have shown that $i_{0} \in J_{*}(\bar{s}) \backslash J_{*}(\hat{s}) \Longrightarrow i_{0} \in J(\bar{s}) \backslash J(\hat{s})$.

In a similar way, we can show that $j_{0} \in J_{*}(\hat{s}) \backslash J_{*}(\bar{s}) \Longrightarrow j_{0} \in J(\hat{s}) \backslash J(\bar{s})$.

Hence, due to the condition (c) (see also (c1)), for $i_{0} \in J_{*}(\bar{s}) \backslash J_{*}(\hat{s}) \subset J_{*}$ and $j_{0} \in J_{*}(\hat{s}) \backslash J_{*}(\bar{s}) \subset J_{*}$, there exists $\bar{D} \in \mathcal{K}$ such that

$$
\left(\boldsymbol{\tau}\left(i_{0}\right)\right)^{\top} \bar{D} \boldsymbol{\tau}\left(j_{0}\right)>0 .
$$

As per the properties of minimal zeroes, it follows that

$$
\boldsymbol{\tau}^{\top} D \boldsymbol{\tau}(j) \geq 0 \forall \boldsymbol{\tau} \in T, \forall j \in J, \forall D \in \mathcal{K} .
$$

Taking into account these inequalities together with (35) and (32), we get

$$
\mathbf{t}^{\top} \bar{D} \mathbf{t}=\sum_{i \in J_{*}} \sum_{j \in J_{*}} \alpha_{j} \alpha_{i}(\boldsymbol{\tau}(j))^{\top} \bar{D} \boldsymbol{\tau}(i) \geq \alpha_{j_{0}} \alpha_{i_{0}}\left(\boldsymbol{\tau}\left(j_{0}\right)\right)^{\top} \bar{D} \boldsymbol{\tau}\left(i_{0}\right)>0,
$$

which contradicts the condition $\mathbf{t} \in T_{0}(\mathcal{K})$. Therefore, relations (33) cannot take place.

Hence, we have shown that $T_{0}(\mathcal{K}) \subset \bigcup_{s \in S} T_{0}(s, \mathcal{K})$. The obtained inclusion and inclusion (31) imply (29).

Remark 3. Note that it follows from Proposition 3 that the set $T_{0}(\mathcal{K})$ can be explicitly described using only the available data (28) (the set of minimal zeros of $\mathcal{K}$ and the set $\mathcal{M}$ ).

Using the partition $\{J(s), s \in S\}$ of the set $J$ satisfying the conditions (a)-(c), let us define the following index sets:

$$
\begin{gathered}
P_{*}(s):=\bigcup_{j \in J(s)} \operatorname{supp}(\boldsymbol{\tau}(j)), s \in S \\
S(j):=\{s \in S: j \in J(s)\}, M_{*}(j):=\bigcup_{s \in S(j)} P_{*}(s), j \in J .
\end{gathered}
$$

Proposition 4. Under the conditions of Proposition 3, the following inclusions hold true:

$$
M_{*}(j) \subset M(j), j \in J,
$$

where the sets $M(j), j \in J$, are defined in (10) and $M_{*}(j), j \in J$, are defined in (37).

Proof. Let us, first, prove the inclusions

$$
P_{*}(s) \subset M(j), s \in S(j), j \in J,
$$

where the sets $P_{*}(s), s \in S(j), j \in J$, are defined in (36) and (37).

Suppose the contrary: for some $j_{0} \in J$ and $s_{0} \in S\left(j_{0}\right)$, it holds $P_{*}\left(s_{0}\right) \not \subset M\left(j_{0}\right)$. This implies the existence of an index $i_{0} \in J\left(s_{0}\right)$ such that $\operatorname{supp}\left(\tau\left(i_{0}\right)\right) \not \subset M\left(j_{0}\right)$. Consequently, there exists $k_{0} \in \operatorname{supp}\left(\tau\left(i_{0}\right)\right)$ such that $k_{0} \notin M\left(j_{0}\right)$. The latter condition implies that there exists a matrix $\bar{D} \in \mathcal{K}$ such that $\mathbf{e}_{k_{0}}^{\top} \bar{D} \boldsymbol{\tau}\left(j_{0}\right)>0$. Furthermore, note that the inclusion 
$s_{0} \in S\left(j_{0}\right)$ implies $j_{0} \in J\left(s_{0}\right)$. Since $i_{0} \in J\left(s_{0}\right)$ and $j_{0} \in J\left(s_{0}\right)$, then by construction, we have $\left(\boldsymbol{\tau}\left(i_{0}\right)\right)^{\top} D \boldsymbol{\tau}\left(j_{0}\right)=0$ for all $D \in \mathcal{K}$. Hence

$$
0=\left(\boldsymbol{\tau}\left(i_{0}\right)\right)^{\top} \bar{D} \boldsymbol{\tau}\left(j_{0}\right)=\sum_{k \in \operatorname{supp}\left(\boldsymbol{\tau}\left(i_{0}\right)\right)} \tau_{k}\left(i_{0}\right) \mathbf{e}_{k}^{\top} \bar{D} \boldsymbol{\tau}\left(j_{0}\right) \geq \tau_{k_{0}}\left(i_{0}\right) \mathbf{e}_{k_{0}}^{\top} \bar{D} \boldsymbol{\tau}\left(j_{0}\right)>0 .
$$

The obtained contradiction proves inclusions (39).

It is evident that inclusions (38) follow from (39) and the definition of the sets $M_{*}(j), j \in$ $J$ (see (37)). The proposition is proved.

Let $\{J(s), s \in S\}$ be the partition of the set $J$ introduced above. Denoted by

$$
\boldsymbol{\tau}_{*}(s):=\frac{1}{|J(s)|} \sum_{j \in J(s)} \boldsymbol{\tau}(j), \quad \overline{\boldsymbol{\tau}}(j):=0.5\left(\boldsymbol{\tau}(j)+\boldsymbol{\tau}_{*}(s)\right), j \in J(s), s \in S .
$$

For any $s \in S$, by construction, we have

$$
\overline{\boldsymbol{\tau}}(j) \in T_{0}(s, \mathcal{K}), \operatorname{supp}(\overline{\boldsymbol{\tau}}(j))=P_{*}(s), j \in J(s) .
$$

Let us prove the following Lemma.

Lemma 6. First, let us show that for any $s \in S$, we have

$$
\begin{aligned}
K_{1}(s): & =\left\{D \in \mathcal{C O} \mathcal{P}^{p}: \mathbf{e}_{k}^{\top} D \overline{\boldsymbol{\tau}}(j)=0, k \in \operatorname{supp}(\overline{\boldsymbol{\tau}}(j)), j \in J(s)\right\} \\
& =\left\{D \in \mathcal{C O P}^{p}: \mathbf{e}_{k}^{\top} D \boldsymbol{\tau}(j)=0, k \in P_{*}(s), j \in J(s)\right\}=: K_{2}(s),
\end{aligned}
$$

where the sets $P_{*}(s), s \in S$, are defined in (36).

Consider any $s \in S$. Recall that the vectors $\overline{\boldsymbol{\tau}}(j), j \in J(s)$, are defined in (40). It follows from these definitions that

$$
\overline{\boldsymbol{\tau}}(j)=\sum_{i \in J(s)} \alpha_{i}(j) \boldsymbol{\tau}(i), \boldsymbol{\tau}(j)=\sum_{i \in J(s)} \beta_{i}(j) \overline{\boldsymbol{\tau}}(i) \forall j \in J(s),
$$

where

$$
\begin{gathered}
\alpha_{*}=\alpha_{*}(s):=\frac{1}{2|J(s)|}, \alpha_{i}(j)=\alpha_{*}, i \in J(s) \backslash j, \alpha_{j}(j)=\alpha_{*}+0.5 ; \\
\beta_{i}(j)=-2 \alpha_{*}, i \in J(s) \backslash j, \beta_{j}(j)=2\left(1-\alpha_{*}\right) \forall j \in J(s) .
\end{gathered}
$$

Notice that, by construction (see (41)), $\operatorname{supp}(\overline{\boldsymbol{\tau}}(j))=P_{*}(s), j \in J(s)$.

Suppose that $D \in K_{1}(s)$. Then, for all $j \in J(s)$ and all $k \in P_{*}(s)$, we have

$$
\mathbf{e}_{k}^{\top} D \boldsymbol{\tau}(j)=\mathbf{e}_{k}^{\top} D \sum_{i \in J(s)} \beta_{i}(j) \overline{\boldsymbol{\tau}}(i)=0
$$

and hence, $D \in K_{2}(s)$.

Now, suppose that $D \in K_{2}(s)$. Then for all $j \in J(s)$ and $k \in P_{*}(s)$, we have

$$
\mathbf{e}_{k}^{\top} D \overline{\boldsymbol{\tau}}(j)=\mathbf{e}_{k}^{\top} D \sum_{i \in J(s)} \alpha_{i}(j) \boldsymbol{\tau}(i)=0,
$$

wherefrom $D \in K_{1}(s)$. Relations (42) are proved.

By construction, $K_{1}=\bigcap_{s \in S} K_{1}(s)$ and it follows from (42) that $K_{1}=\bigcap_{s \in S} K_{2}(s)$.

Suppose that $D \in K_{1}=\bigcap_{s \in S} K_{2}(s)$. Hence, for any $j \in J$, we have $\mathbf{e}_{k}^{\top} D \boldsymbol{\tau}(j)=0 \forall k \in P_{*}(s)$, $\forall s \in S(j)$. These equalities and (37) imply the equalities $\mathbf{e}_{k}^{\top} D \boldsymbol{\tau}(j)=0, k \in M_{*}(j), j \in J$. Then, by definition, $D \in K_{2}$. 
Now let $D \in K_{2}$. Taking into account (37), we conclude that $\mathbf{e}_{k}^{\top} D \boldsymbol{\tau}(j)=0, k \in P_{*}(s), j \in$ $J(s), s \in S$. Consequently, $D \in \bigcap_{s \in S} K_{2}(s)=K_{1}$. The lemma is proved.

Note that it follows from Proposition 2 that the cone $K_{1}$ is an exposed face of $\mathcal{C O P}{ }^{p}$ and therefore, from the lemma above it follows that the cone $K_{2}$ is an exposed face of $\mathcal{C O P}{ }^{p}$ as well.

Theorem 2. Let $\mathcal{K}$ be a face of the cone $\mathcal{C O P}^{p}$. Consider the corresponding finite data sets (28) and the sets $M_{*}(j), j \in J$, defined in (37). Then the set

$$
\mathcal{K}_{\text {exp }}^{\min }:=\left\{D \in \mathcal{C O} \mathcal{P}^{p}: \mathbf{e}_{k}^{\top} D \boldsymbol{\tau}(j)=0, k \in M_{*}(j), j \in J\right\}
$$

is the minimal exposed face containing the face $\mathcal{K}$.

Proof. Note that it follows from (38) that $\mathcal{K} \subset \mathcal{K}_{\text {exp }}^{\min }$. By construction, $\mathcal{K}_{\text {exp }}^{\min }=K_{2}$, where $K_{2}$ is an exposed face. Then $\mathcal{K}_{\text {exp }}^{\min }$ is an exposed face of $\mathcal{C O P}^{p}$ containing the face $\mathcal{K}$.

To prove that $\mathcal{K}_{\text {exp }}^{\min }$ is the minimal exposed face, suppose the contrary: there exists another exposed face $K_{\text {exp }}$, such that $\mathcal{K} \subset K_{\exp } \subset \mathcal{K}_{\text {exp }}^{\min }=K_{1}=K_{2}$ and a matrix $\bar{D} \in \mathcal{K}_{\text {exp }}^{\min }$, such that $\bar{D} \notin K_{\text {exp }}$.

As $K_{\text {exp }}$ is an exposed face, for some vectors $\xi(i) \in T, i \in I$, it holds

$$
K_{\exp }=\left\{D \in \mathcal{C O P}^{p}:(\xi(i))^{\top} D \xi(i)=0, i \in I\right\},|I|<\infty .
$$

Since $\bar{D} \notin K_{\text {exp }}$, then there exists $i_{0} \in I$ such that $\left(\mathcal{S}\left(i_{0}\right)\right)^{\top} \bar{D} \mathcal{\xi}\left(i_{0}\right)>0$. By assumption, $\mathcal{K} \subset K_{\exp }$. Then $\boldsymbol{\xi}\left(i_{0}\right) \in T_{0}(\mathcal{K})$ and, consequently $\boldsymbol{\xi}\left(i_{0}\right) \in T_{0}\left(s_{0}, \mathcal{K}\right)$ for some $s_{0} \in S$, where the set $T_{0}\left(s_{0}, \mathcal{K}\right)$ is defined in (30). Hence $\boldsymbol{\xi}\left(i_{0}\right)=\sum_{j \in J\left(s_{0}\right)} \alpha_{j} \tau(j)$ with some $\alpha_{j} \geq 0, j \in J\left(s_{0}\right)$, $\sum_{j \in J\left(s_{0}\right)} \alpha_{j}=1$. These relations together with (43) imply

$$
\boldsymbol{\xi}\left(i_{0}\right)=\sum_{j \in J\left(s_{0}\right)} \alpha_{j} \sum_{i \in J\left(s_{0}\right)} \beta_{i}(j) \overline{\boldsymbol{\tau}}(i)=\sum_{i \in J\left(s_{0}\right)} \beta_{i} \overline{\boldsymbol{\tau}}(i) ; \operatorname{supp}\left(\boldsymbol{\xi}\left(i_{0}\right)\right) \subset P_{*}\left(s_{0}\right),
$$

where $\beta_{i}=2\left(\alpha_{i}-\alpha_{*}\left(s_{0}\right)\right), i \in J\left(s_{0}\right), \alpha_{*}\left(s_{0}\right)=1 /\left|J\left(s_{0}\right)\right|$.

From the assumption $\bar{D} \in \mathcal{K}_{\text {exp }}^{\min }=K_{1}$, it follows $\bar{D} \in K_{1}(s) \forall s \in S$ (where the sets $K_{1}(s), s \in S$, are defined in (42)) and hence, $\bar{D} \in K_{1}\left(s_{0}\right)$.

Taking into account the latter inclusion and relations (46), we get

$$
\left(\boldsymbol{\xi}\left(i_{0}\right)\right)^{\top} \bar{D} \boldsymbol{\xi}\left(i_{0}\right)=\left(\boldsymbol{\xi}\left(i_{0}\right)\right)^{\top} \bar{D} \sum_{j \in J\left(s_{0}\right)} \beta_{j} \overline{\boldsymbol{\tau}}(j)=\sum_{k \in \operatorname{supp}\left(\boldsymbol{\xi}\left(i_{0}\right)\right)} \xi_{k}\left(i_{0}\right) \mathbf{e}_{k}^{\top} \bar{D} \sum_{j \in J\left(s_{0}\right)} \beta_{j} \overline{\boldsymbol{\tau}}(j)=0 .
$$

This result contradicts the inequality $\left(\boldsymbol{\xi}\left(i_{0}\right)\right)^{\top} \bar{D} \boldsymbol{\zeta}\left(i_{0}\right)>0$, which proves the theorem.

Using Theorem 2 and following the proof of Lemma 4, one can easily prove the following statement.

Corollary 2. Any face of the cone $\mathrm{COP}^{p}$ is an exposed face of its minimal exposed face.

Note that $T_{0}(\mathcal{K})=T_{0}\left(\mathcal{K}_{\text {exp }}^{\min }\right)$ for any face $\mathcal{K}$ and the corresponding minimal exposed face $\mathcal{K}_{\text {exp }}^{\min }$. 
Theorem 3. A face $\mathcal{K} \triangleleft \mathcal{C O P}^{p}$ is exposed if and only if $W=W_{*}$, where

$$
\begin{aligned}
& W:=\left\{D \in \mathcal{S}^{p}: \mathbf{e}_{k}^{\top} D \boldsymbol{\tau}(j)=0, k \in M(j), \mathbf{e}_{k}^{\top} D \boldsymbol{\tau}(j) \geq 0, k \in[p] \backslash M(j), j \in J\right\}, \\
& W_{*}:=\left\{D \in \mathcal{S}^{p}: \mathbf{e}_{k}^{\top} D \boldsymbol{\tau}(j)=0, k \in M_{*}(j), \mathbf{e}_{k}^{\top} D \boldsymbol{\tau}(j) \geq 0, k \in[p] \backslash M_{*}(j), j \in J\right\} .
\end{aligned}
$$

Proof. Let us show that the faces $\mathcal{K}$ and $\mathcal{K}_{\text {exp }}^{\min }$ of $\mathcal{C O P}{ }^{p}$ admit representations

$$
\mathcal{K}=\left\{D \in W: \mathbf{t}^{\top} D \mathbf{t} \geq 0 \forall \mathbf{t} \in \Omega\left(V_{0}\right)\right\}, \mathcal{K}_{\text {exp }}^{\min }=\left\{D \in W_{*}: \mathbf{t}^{\top} D \mathbf{t} \geq 0 \forall \mathbf{t} \in \Omega\left(V_{0}\right)\right\},
$$

where $V_{0}$ is the set of all minimal zeros of these faces, and the set $\Omega\left(V_{0}\right)$ is defined in (4).

Let $\mathcal{K}$ be a face of $\mathcal{C O P}{ }^{p}$ with the corresponding set of minimal zeros $V_{0}=\{\boldsymbol{\tau}(j), j \in J\}$. Let the set $\mathcal{M}=\{M(j), j \in J\}$ be defined in (11). Then, according to Lemma 3 (see (12)), the face $\mathcal{K}$ can be presented in the form

$$
\mathcal{K}=\left\{D \in \mathcal{C O} \mathcal{P}^{p}: \mathbf{e}_{k}^{\top} D \boldsymbol{\tau}(j)=0, k \in M(j), \mathbf{e}_{k}^{\top} D \boldsymbol{\tau}(j) \geq 0, k \in[p] \backslash M(j), j \in J\right\} .
$$

Taking into account the definitions of the cone $\mathcal{C O P}^{p}$ and the set $W$ (see (47)), we obtain

$$
\mathcal{K}=\left\{D \in W: \mathbf{t}^{\top} D \mathbf{t} \geq 0 \forall \mathbf{t} \in T\right\}
$$

Now consider the cone

$$
\overline{\mathcal{K}}:=\left\{D \in W: \mathbf{t}^{\top} D \mathbf{t} \geq 0 \forall \mathbf{t} \in \Omega\left(V_{0}\right)\right\} .
$$

Since $\Omega\left(V_{0}\right) \subset T$ then it is evident that $\mathcal{K} \subset \overline{\mathcal{K}}$.

Let us show that $\overline{\mathcal{K}} \subset \mathcal{K}$. Consider any $D \in \overline{\mathcal{K}}$. It is evident that the conditions $D \in W, \mathbf{t}^{\top} D \mathbf{t} \geq 0 \forall \mathbf{t} \in \Omega\left(V_{0}\right)$ imply that $D \boldsymbol{\tau}(j) \geq 0 \forall j \in J, \mathbf{t}^{\top} D \mathbf{t} \geq 0 \forall \mathbf{t} \in \Omega\left(V_{0}\right)$ and due to Lemma 1 we conclude that $D \in \mathcal{C O O P}{ }^{p}$. This implies that $D \in \mathcal{K}$. Thus, we have proved that the cone $\mathcal{K}=\mathcal{K}\left(V_{0}, \mathcal{M}\right)$ can be presented as $\overline{\mathcal{K}}$.

Similarly, it can be shown that the cone $\mathcal{K}_{\text {exp }}^{\min }$ defined in (44) can be presented in the form (48) with $W_{*}$ defined in (47). Thus, representations (48) are justified.

As before, based on (14) and (18), it is simple to show that there exists $B \in \mathcal{K}_{e x p}^{\min }$ such that $\mathbf{t}^{\top} B \mathbf{t}>0 \quad \forall \mathbf{t} \in \Omega\left(V_{0}\right)$. Note that the set $\Omega\left(V_{0}\right)$ is closed and bounded; therefore, for some sufficiently small $\mu>0$, it holds

$$
\mathbf{t}^{\top} B \mathbf{t}>\mu \forall \mathbf{t} \in \Omega\left(V_{0}\right) .
$$

Note that, from (38), it follows $W \subset W_{*}$. Suppose that $W \neq W_{*}$. Then there exists $\bar{D} \in W_{*}$ such that $\bar{D} \notin W$. From the conditions $B \in \mathcal{K}_{\text {exp }}^{\min }, \bar{D} \in W_{*}, \bar{D} \notin W$, and inequalities (49), it follows that for a sufficiently small $\delta>0$, we have

$$
\mathbf{t}^{\top}(B+\delta \bar{D}) \mathbf{t} \geq 0 \forall \mathbf{t} \in \Omega\left(V_{0}\right) ; \quad(B+\delta \bar{D}) \in W_{*}, \quad(B+\delta \bar{D}) \notin W .
$$

Taking into account (48), we conclude that $(B+\delta \bar{D}) \notin \mathcal{K}$ and $(B+\delta \bar{D}) \in \mathcal{K}_{\text {exp }}^{\min }$. Therefore $\mathcal{K} \neq \mathcal{K}_{\text {exp }}^{\min }$. As $\mathcal{K}_{\text {exp }}^{\min }$ is the minimal exposed face containing $\mathcal{K}$, it is obvious that $\mathcal{K}$ is not exposed.

Now, suppose that $W=W_{*}$. Then it follows from (48) that $\mathcal{K}=\mathcal{K}_{\text {exp }}^{\min }$ and hence $\mathcal{K}$ is an exposed face. The theorem is proved.

Theorem 3 reduces the answer to the question of whether a given face is exposed to checking the condition $W=W_{*}$, which can be done by solving a unique LP problem when the data (28) is assumed to be known.

Remark 4. Note that, given a face $\mathcal{K} \unlhd \mathcal{C O P}{ }^{p}$, one can show that the respective minimal zeros $\tau(j)$ and the sets $M_{*}(j), j \in J$, can be uniquely constructed using only the set $T_{0}(\mathcal{K})$ of all zeros of 
this face. Hence the minimal exposed face containing $\mathcal{K}$ can be constructed on the basis of the set of all zeros of $\mathcal{K}$.

There may exist several different faces $\mathcal{K}^{m}, m=1, \ldots, m_{0}, m_{0}>1$, having the same sets of all their zeros: $T_{0}\left(\mathcal{K}^{l}\right)=T_{0}\left(\mathcal{K}^{m}\right) \forall l, m \in\left[m_{0}\right]$. Hence all these faces have the same minimal exposed face.

Let us illustrate this with the following example.

Example 3. For $p=3$, consider the sets

$$
\begin{gathered}
V^{1}=\left\{\mathbf{t}(1)=\mathbf{e}_{1}, \mathbf{t}(2)=\mathbf{e}_{3}\right\}, \mathcal{L}^{1}=\left\{L^{1}(1)=\{1,2,3\}, L^{1}(2)=\{1,3\}\right\}, \\
V^{2}=V^{1}, \mathcal{L}^{2}=\left\{L^{2}(1)=\{1,3\}, L^{2}(2)=\{1,2,3\}\right\}, \\
V^{3}=\left\{\mathbf{t}(1)=0.5\left(\mathbf{e}_{1}+\mathbf{e}_{3}\right), \mathbf{t}(2)=\mathbf{e}_{1}\right\}, \mathcal{L}^{3}=\left\{L^{3}(1)=\{1,3\}, L^{3}(2)=\{1\}\right\} .
\end{gathered}
$$

It is easy to check that the faces $\mathcal{K}^{i}:=\mathcal{K}\left(V^{i}, \mathcal{L}^{i}\right), i=1,2,3$, are as follows:

$$
\mathcal{K}^{1}=\{D \in \mathbf{K}: b=0\}, \mathcal{K}^{2}=\{D \in \mathbf{K}: f=0\}, \mathcal{K}^{3}=\mathbf{K},
$$

where the face $\mathbf{K}$ is defined in (15).

For each face $\mathcal{K}^{m}, m=1,2,3$, denote by $T_{0}\left(\mathcal{K}^{m}\right)$ the corresponding set of zeros, by $V_{0}^{m}=$ $\left\{\boldsymbol{\tau}^{m}(j), j \in J^{m}\right\}$ the set of minimal zeros, and by $M^{m}(j), M_{*}^{m}(j), j \in J^{m}$, the sets defined in (17), (37), respectively. Here we have

$$
\begin{aligned}
& T_{0}\left(\mathcal{K}^{m}\right)=T_{0}(*):=\left\{\mathbf{t}=\alpha a \mathbf{e}_{1}+(1-\alpha) \mathbf{e}_{3}, \forall \alpha \in[0,1]\right\}, \\
& V_{0}\left(\mathcal{K}^{m}\right)=\left\{\boldsymbol{\tau}^{m}(1)=\mathbf{e}_{1}, \boldsymbol{\tau}^{m}(2)=\mathbf{e}_{3}\right\}, J^{m}=\{1,2\}, \\
& M_{*}^{m}(1)=M_{*}^{m}(2)=\{1,3\}, m=1,2,3 ; \\
& M^{m}(j)=L^{m}(j) \forall j \in J^{m}, m=1,2, M^{3}(j)=M_{*}^{3}(j) \forall j \in J^{3} .
\end{aligned}
$$

For $m=1,2$, the faces $\mathcal{K}^{m}$, are not exposed since $W^{m} \neq W_{*}^{m}$, and the face $\mathcal{K}^{3}$ is exposed by construction (see Proposition 2). Here the sets $W^{m}$ and $W_{*}^{m}$ are defined by (47) for $\mathcal{K}^{m}$.

Note that the faces $\mathcal{K}^{m}, m=1,2,3$, are different, but have the same set $T_{0}(*)$ of zeros. Hence for any $\mathcal{K}^{m}, m=1,2,3$, the minimal exposed face $\mathcal{K}_{\text {exp }}^{\min }(m)$ containing $\mathcal{K}^{m}$ should be the same. In fact, in our case, for $m=1,2,3$, we have $\mathcal{K}_{\text {exp }}^{\min }(m)=\mathcal{K}^{3}$.

\subsection{The Minimal and the Minimal Exposed Faces Containing a Given Set}

Based on the results obtained in Section 3.2 and in [15], it is easy to describe the minimal exposed face containing a given closed convex subset of $\mathcal{C O} \mathcal{P}^{p}$.

Let $Q$ be a convex subset of $\mathcal{C O P}{ }^{p}$. Consider the corresponding set of zeros

$$
T_{0}(Q):=\left\{\mathbf{t} \in T: \mathbf{t}^{\top} D \mathbf{t}=0 \forall D \in Q\right\},
$$

that is either empty or the union of a finite number of convex bounded polyhedra.

Let $V_{0}(Q)=\left\{\boldsymbol{\tau}(i), i \in J_{Q}\right\}$ be the set of all vertices of the set $\operatorname{conv} T_{0}(Q)$.

It was shown in [15] that the minimal face of $\mathcal{C O P}{ }^{p}$ containing $Q$ can be presented in the form $\mathcal{K}\left(V_{0}(Q), \mathcal{M}(Q)\right)$, where

$$
\mathcal{M}(Q):=\left\{M_{Q}(j), j \in J_{Q}\right\}, M_{Q}(j):=\left\{k \in[p]: \mathbf{e}_{k}^{\top} D \boldsymbol{\tau}(j)=0 \quad \forall D \in Q\right\}, j \in J_{Q} .
$$

Applying Theorem 2, we conclude that the minimal exposed face containing the set $Q$ has the form

$$
\mathcal{K}_{\text {exp }}^{\min }(Q)=\left\{D \in \mathcal{C O} \mathcal{P}^{p}: \mathbf{e}_{k}^{\top} D \boldsymbol{\tau}(j)=0, k \in M_{* Q}(j), j \in J_{Q}\right\},
$$


where the sets $M_{* Q}(j), j \in J_{Q}$, are defined by the rules (37) with the sets $V_{0}$ and $\mathcal{M}$ replaced by $V_{0}(Q)$ and $\mathcal{M}(Q)$.

\section{Faces of $\mathcal{C O P}{ }^{p}$ Defined by Singleton Sets}

In this section, we consider a special class of faces of $\mathcal{C O P}{ }^{p}$, namely, faces $\mathcal{K}=\mathcal{K}(V, \mathcal{L})$ defined by the sets $V$ and $\mathcal{L}$, where $V$ is a singleton. Since $V$ is a singleton, then by construction, the set $\mathcal{L}$ is a singleton as well and we can assume that these sets have the form

$$
V=\{\boldsymbol{\tau}\} \subset T, \mathcal{L}=\{L\}, \text { where } L \text { is such that } \operatorname{supp}(\boldsymbol{\tau}) \subset L \subset[p] .
$$

Note that the class of faces defined by singleton sets is interesting in terms of studying the facial structure of the cone $\mathcal{C O P}^{p}$ since all maximal faces of $\mathcal{C O P}{ }^{p}$ belong to it.

Recall that by definition, a face $\mathcal{F}$ of $\mathcal{C O P}{ }^{p}$ is the maximal face if $\mathcal{F} \neq \mathcal{C O P}{ }^{p}$ and there does not exist a face $\overline{\mathcal{F}} \neq \mathcal{C O} \mathcal{P}^{p}$ such that $\overline{\mathcal{F}} \neq \mathcal{F}$ and $\mathcal{F} \subset \overline{\mathcal{F}}$.

The next lemma is proved in [11].

Lemma 7. A face $\mathcal{F} \unlhd \mathcal{C O P}{ }^{p}$ is maximal if and only if there exists $\tau \in T$ such that $\mathcal{F}=\{D \in$ $\left.\mathcal{C O P}^{p}: \boldsymbol{\tau}^{\top} D \boldsymbol{\tau}=0\right\}$.

The main result of this section is the proof of the following lemma.

Lemma 8. Consider a face $\mathcal{K}=\mathcal{K}(V, \mathcal{L})$ with the defining sets $V$ and $\mathcal{L}$ satisfying (50). Then

(i) $T_{0}(\mathcal{K}(V, \mathcal{L}))=V$ and $\mathcal{M}:=\left\{k \in[p]: \mathbf{e}_{k}^{\top} D \boldsymbol{\tau}=0 \forall D \in \mathcal{K}(V, \mathcal{L})\right\}=L$; $\mathcal{K}(\bar{V}, \overline{\mathcal{L}})$;

(ii) the pair $\{V, \mathcal{L}\}$ is unique, i.e., there is no other pair $\{\bar{V}, \overline{\mathcal{L}}\}$ such that $\mathcal{K}(V, \mathcal{L})=$

(iii) $\operatorname{dim}(\mathcal{K}(V, \mathcal{L}))=p(p+1) / 2-|L|$.

Proof. (i) Let us show that $T_{0}(\mathcal{K}(V, \mathcal{L}))=\{\boldsymbol{\tau}\}$ and $\mathcal{M}=L$. Without loss of generality, we can suppose that

$$
\operatorname{supp}(\boldsymbol{\tau})=\left[q_{*}\right], L=[q], 1 \leq q_{*} \leq q \leq p,
$$

and present $\boldsymbol{\tau}$ in the form $\boldsymbol{\tau}^{\top}=\left(\boldsymbol{\tau}_{*}^{\top}, \mathbf{0}_{p-q}^{\top}\right)$, where $\boldsymbol{\tau}_{*}^{\top}=\left(\tau_{1}, \ldots, \tau_{q}\right)$ with $\tau_{k}>0, k=$ $1, \ldots, q_{*}$, and $\mathbf{0}_{p-q}^{\top}=(0, \ldots, 0) \in \mathbb{R}^{p-q}$.

Let $\left\{\boldsymbol{\tau}_{*}, \boldsymbol{\varphi}_{k}, k=1, \ldots, q-1\right\}$ be an orthogonal basis in $\mathbb{R}^{q}$. Denoted by

$$
\Phi:=\left(\varphi_{k}, k=1, \ldots, q-1\right) \in \mathbb{R}^{q \times(q-1)}, D_{*}:=\Phi(\Phi)^{\top} \in \mathbb{R}^{q \times q} .
$$

Consider any vector $\mathbf{t}_{*} \in \mathbb{R}^{q}$. Since the representation $\mathbf{t}_{*}=\sum_{k=1}^{q-1} \alpha_{k} \boldsymbol{\varphi}_{k}+\alpha_{*} \boldsymbol{\tau}_{*}$ holds, it is easy to check that

$$
\mathbf{t}_{*}^{\top} D_{*} \mathbf{t}_{*}=\sum_{k=1}^{q-1} \alpha_{k}^{2}\left\{\begin{array}{l}
>0 \text { for all } \mathbf{t}_{*} \in \mathbb{R}^{q} \backslash\{\boldsymbol{\tau}\},\left\|\mathbf{t}_{*}\right\|=1, \\
=0 \text { for } \mathbf{t}_{*}=\boldsymbol{\tau} .
\end{array}\right.
$$

Consider a matrix $\bar{D}:=\left(\begin{array}{cc}D_{*} & D_{0} \\ D_{0}^{\top} & I\end{array}\right) \in \mathbb{R}^{p \times p}$, where $I$ is the $(p-q) \times(p-q)$ identity matrix and $D_{0}$ is a $q \times(p-q)$ matrix with positive elements.

By construction, $\mathbf{t}^{\top} \bar{D} \mathbf{t}>0 \forall \mathbf{t} \in T \backslash\{\boldsymbol{\tau}\}$ and $\boldsymbol{\tau}^{\top} \bar{D} \boldsymbol{\tau}=0$, and

$$
\mathbf{e}_{k}^{\top} \bar{D} \boldsymbol{\tau}=0 \forall k \in L, \mathbf{e}_{k}^{\top} \bar{D} \boldsymbol{\tau}>0 \forall k \in[p] \backslash L .
$$

Then $\bar{D} \in \mathcal{K}(V, \mathcal{L}), T_{0}(\mathcal{K}(V, \mathcal{L}))=\{\boldsymbol{\tau}\}$, and $\mathcal{M}=L$.

(ii) Using the latter equalities, it is easy to show that the face $\mathcal{K}=\mathcal{K}(V, \mathcal{L})$ has a unique defining pair $\{V, \mathcal{L}\}$, i.e., there no other sets $\bar{V}$ and $\overline{\mathcal{L}}$ such that $\mathcal{K}(V, \mathcal{L})=\mathcal{K}(\bar{V}, \overline{\mathcal{L}})$. 
(iii) Finally, let us prove that the dimension of the face $\mathcal{K}=\mathcal{K}(V, \mathcal{L})$ with $V=\{\boldsymbol{\tau}\}$, $\tau \in T$, is equal to $p(p+1) / 2-|L|$. Taking into account Corollary 1 , it is enough to show that

$$
\operatorname{dim} \mathcal{H}(V, \mathcal{L})=\operatorname{dim}\left\{D \in \mathcal{S}^{p}: D \bullet\left(\mathbf{e}_{k} \boldsymbol{\tau}^{\top}+\boldsymbol{\tau} \mathbf{e}_{k}^{\top}\right)=0, k \in L\right\}=p(p+1) / 2-|L| .
$$

Let us prove that the matrices

$$
\left(\mathbf{e}_{k} \boldsymbol{\tau}^{\top}+\boldsymbol{\tau} \mathbf{e}_{k}^{\top}\right), k \in L,
$$

are linearly independent. Suppose the contrary, i.e., there exist $y_{k}, k \in L$, such that

$$
\sum_{k \in L} y_{k}\left(\mathbf{e}_{k} \boldsymbol{\tau}^{\top}+\boldsymbol{\tau} \mathbf{e}_{k}^{\top}\right)=\mathbb{O}_{p \times p}, \sum_{k \in L}\left|y_{k}\right|>0 .
$$

The equality in (53) implies $y_{k} \tau_{s}+y_{s} \tau_{k}=0 \forall k \in L, \forall s \in L$ and hence $y_{k}=0 \forall k \in L$, which contradicts the inequality in (53). Thus, the matrices in (52) are linearly independent and therefore $\operatorname{dim} \mathcal{K}(V, \mathcal{L})=p(p+1) / 2-|L|$.

Note that the above-proven lemma generalizes the related result from [11], where it was shown that a maximal face $\mathcal{K}^{\text {max }}:=\left\{D \in \mathcal{C O} \mathcal{P}^{p}: \tau^{\top} D \tau=0\right\}$ of $\mathcal{C O P}^{p}$ has dimension $\operatorname{dim}\left(\mathcal{K}^{\max }\right)=(p+1) p / 2-|\operatorname{supp}(\boldsymbol{\tau})|$.

For a given $\boldsymbol{\tau} \in T$, set $m_{0}=2^{(p-|\operatorname{supp}(\boldsymbol{\tau})|)}$ and consider faces

$$
\mathcal{K}^{m}=\mathcal{K}\left(V, \mathcal{L}^{m}\right), m=1, \ldots, m_{0},
$$

with the defining sets $V=\{\boldsymbol{\tau}\}$ and $\mathcal{L}^{m}=\left\{L^{m}\right\}$ such that $\operatorname{supp}(\boldsymbol{\tau}) \subset L^{m} \subset[p]$, and $L^{m} \neq L^{\bar{m}}$, for all $m \neq \bar{m}$.

It follows from Lemma 8 that all faces in (54) are different; for any $m=1, \ldots, m_{0}$, the face $\mathcal{K}^{m}$ has a unique pair of defining sets $V=\{\boldsymbol{\tau}\}$ and $\mathcal{L}^{m}$, and its dimension is $\operatorname{dim}\left(\mathcal{K}^{m}\right)=p(p+1) / 2-\left|L^{m}\right|$.

Set $\mathcal{L}^{1}:=\{\operatorname{supp}(\tau)\}$. From Theorem 2 , one can conclude that $\mathcal{K}\left(V, \mathcal{L}^{1}\right)$ is the minimal exposed face of $\mathcal{C O P}{ }^{p}$ containing all other faces $\mathcal{K}^{m}, m=2, \ldots, m_{0}$, which are all not exposed. Note that if $\operatorname{supp}(\boldsymbol{\tau})=[p]$, then $m_{0}=1$ and the set of faces defined in (54) consists of a unique exposed face $\mathcal{K}\left(V, \mathcal{L}^{1}\right)$.

\section{Examples}

In this section, we present a few more examples that justify the advantages of the approach developed here to the study of the facial structure of the cone $\mathcal{C O P}{ }^{p}$ and illustrate some of the properties of the faces of $\mathcal{C O P}{ }^{p}$.

Let us start with an example illustrating the usefulness of the set of minimal zeros of a face of the cone $\mathcal{C O P}{ }^{p}$ and the corresponding set $\mathcal{M}$, introduced in this paper. In particular, this example shows that these sets make it possible to explicitly describe the cone dual to this face without using the closure operator.

As above, for some sets $V$ and $\mathcal{L}$ in the form (2) and (7), we will consider face $\mathcal{K}=\mathcal{K}(V, \mathcal{L})$ of the cone $\mathcal{C O} \mathcal{P}^{p}$ defined in (5), the corresponding set $V_{0}=\{\boldsymbol{\tau}(j), j \in J\}$ of its minimal zeros, and the set $\mathcal{M}$ of the sets $M(j), j \in J$, defined by rules (17).

Consider the dual to $\mathcal{K}$ cone $\mathcal{K}^{*}$. It was shown in [15] that

$$
\mathcal{K}^{*}=\mathrm{cl} Z_{0}=\mathrm{clZ}_{1}=Z_{2},
$$

where

$$
\begin{aligned}
Z_{0}:=\left\{D \in \mathcal{S}^{p}: D=\sum_{i=1}^{p_{*}} \boldsymbol{\mu}(i)(\boldsymbol{\mu}(i))^{\top}+\sum_{i \in I}\left(\boldsymbol{\lambda}(i)(\mathbf{t}(i))^{\top}+\mathbf{t}(i)(\boldsymbol{\lambda}(i))^{\top}\right),\right. \\
\left.\boldsymbol{\mu}(i) \in \mathbb{R}_{+}^{p}, i=1, \ldots, p_{*}, \lambda(i) \in \mathbb{R}^{p}, \lambda_{k}(i)=0, k \in[p] \backslash L(i), i \in I\right\},
\end{aligned}
$$




$$
\begin{aligned}
Z_{1}:=\left\{D \in \mathcal{S}^{p}: D=\sum_{i=1}^{p_{*}} \boldsymbol{\mu}(i)(\boldsymbol{\mu}(i))^{\top}+\sum_{i \in I}\left(\boldsymbol{\lambda}(i)(\mathbf{t}(i))^{\top}+\mathbf{t}(i)(\boldsymbol{\lambda}(i))^{\top}\right),\right. \\
\left.\boldsymbol{\mu}(i) \in \mathbb{R}_{+}^{p}, i=1, \ldots, p_{*} ; \lambda(i) \in \mathbb{R}^{p} ; \lambda_{k}(i) \geq 0, k \in[p] \backslash L(i), i \in I\right\}, \\
Z_{2}:=\left\{D \in \mathcal{S}^{p}: D=\sum_{i=1}^{p_{*}} \boldsymbol{\mu}(i)(\boldsymbol{\mu}(i))^{\top}+\sum_{j \in J}\left(\boldsymbol{\eta}(j)(\boldsymbol{\tau}(j))^{\top}+\boldsymbol{\tau}(j)(\boldsymbol{\eta}(j))^{\top}\right),\right. \\
\left.\boldsymbol{\mu}(i) \in \mathbb{R}_{+}^{p}, i=1, \ldots, p_{*} ; \boldsymbol{\eta}(j) \in \mathbb{R}^{p} ; \eta_{k}(j) \geq 0, k \in[p] \backslash M(j), j \in J\right\} .
\end{aligned}
$$

Here, $\mathrm{cl} \mathcal{B}$ denotes the closure of a set $\mathcal{B}$.

It was shown in [15] that $Z_{0} \subset Z_{1} \subset Z_{2}$ and, in general, $Z_{0} \neq Z_{1}$.

Now, let us illustrate that in general $Z_{1} \neq Z_{2}$ and, therefore, $\mathcal{K}^{*} \neq Z_{1}$, which means that we cannot omit the use of the closure operator in that equalities in (55) that concern the sets $Z_{0}$ and $Z_{1}$ (constructed using the defining sets $V$ and $\mathcal{L}$ ), while in the equality in (55) regarding the set $Z_{2}$ (constructed using the sets $V_{0}$ and $\mathcal{M}$ ), no closure operator is needed. This gives us an explicit description of the dual cone $\mathcal{K}^{*}$.

Example 4. Let us once again consider Example 1 (see Section 2.2), where $p=3, I=\{1,2\}$, and $\mathbf{t}(1)=0.5\left(\begin{array}{lll}1 & 0 & 1\end{array}\right)^{\top}, \mathbf{t}(2)=\left(\begin{array}{lll}1 & 0 & 0\end{array}\right)^{\top}, V=\{\mathbf{t}(1), \mathbf{t}(2)\}, \mathcal{L}=\{L(1), L(2)\}$, with $L(1)=$ $\{1,3\}, L(2)=\{1\}$. We have shown that the face $\mathcal{K}=\mathcal{K}(V, \mathcal{L})$ of $\mathcal{C O} \mathcal{P}^{3}$ has the form (15) and $V_{0}=\left\{\boldsymbol{\tau}(1)=\mathbf{e}_{1}, \boldsymbol{\tau}(2)=\mathbf{e}_{3}\right\}, J=\{1,2\}, M(1)=M(2)=\{1,3\}$.

Consider a vector $\boldsymbol{\eta}=\left(\eta_{1}, \eta_{2}, \eta_{3}\right)^{\top}$, where $\eta_{1} \geq 0, \eta_{2}>0$, and the matrix

$$
\Theta:=\boldsymbol{\eta}(\boldsymbol{\tau}(2))^{\top}+\boldsymbol{\tau}(2) \boldsymbol{\eta}^{\top}=\left(\begin{array}{ccc}
0 & 0 & \eta_{1} \\
0 & 0 & \eta_{2} \\
\eta_{1} & \eta_{2} & 2 \eta_{3}
\end{array}\right)=\left[\theta_{k, s}\right]_{s=1, \ldots, p ; k=1, \ldots, p} \in \mathcal{S}^{3} .
$$

By construction, $\Theta \in Z_{2}$, and it is easy to see that $D \bullet \Theta=2 \eta_{2} f \geq 0$ for any $D \in \mathcal{K}$. Consequently, $\Theta \in \mathcal{K}^{*}$.

Suppose that $\Theta \in Z_{1}$. Hence it can be presented in the form

$$
\begin{gathered}
\Theta=\sum_{i=1}^{p_{*}} \boldsymbol{\mu}(i)(\boldsymbol{\mu}(i))^{\top}+\sum_{i \in I}\left(\boldsymbol{\lambda}(i)(\mathbf{t}(i))^{\top}+\mathbf{t}(i)(\boldsymbol{\lambda}(i))^{\top}\right) \\
=\Omega+0.5\left(\begin{array}{ccc}
2 \lambda_{1}(1) & \lambda_{2}(1) & \lambda_{1}(1)+\lambda_{3}(1) \\
\lambda_{2}(1) & 0 & \lambda_{2}(1) \\
\lambda_{1}(1)+\lambda_{3}(1) & \lambda_{2}(1) & 2 \lambda_{3}(1)
\end{array}\right)+\left(\begin{array}{ccc}
2 \lambda_{1}(2) & \lambda_{2}(2) & \lambda_{3}(2) \\
\lambda_{2}(2) & 0 & 0 \\
\lambda_{3}(2) & 0 & 0
\end{array}\right),
\end{gathered}
$$

where $\lambda_{2}(1) \geq 0, \lambda_{2}(2) \geq 0, \lambda_{3}(2) \geq 0, \Omega=\sum_{i=1}^{p_{*}} \boldsymbol{\mu}(i)(\boldsymbol{\mu}(i))^{\top}=\left[\omega_{k, s}\right]_{s=1,2,3 ; k=1,2,3}$ with $\omega_{k, s}=\sum_{i=1}^{p_{*}} \mu_{k}(i) \mu_{s}(i) \geq 0, k=1,2,3 ; s=1,2,3$.

It follows from the latest relations that

$$
\begin{gathered}
0=\theta_{2,1}=0.5 \lambda_{2}(1)+\lambda_{2}(2)+\omega_{2,1} \text {, where } \lambda_{2}(1) \geq 0, \lambda_{2}(2) \geq 0, \omega_{2,1} \geq 0, \\
0=\theta_{2,2}=0+0+\omega_{2,2} \text { where } \omega_{2,2} \geq 0, \\
\text { and } 0<\theta_{2,3}=0.5 \lambda_{2}(1)+\omega_{2,3} .
\end{gathered}
$$

From (56) and (57), we get $\lambda_{2}(1)=0, \lambda_{2}(2)=0, \omega_{2,1}=0, \omega_{2,2}=0$. The equality $\omega_{2,2}=0$ implies $\omega_{1,2}=\omega_{2,2}=\omega_{2,3}=\omega_{2,1}=\omega_{3,2}=0$. Then the relation (58) takes the form

$$
0<\theta_{2,3}=\lambda_{2}(1)+\omega_{2,3}=0 \text {. }
$$

The obtained contradiction proves that $\Theta \notin Z_{1}$. Remind that by construction, $\Theta \in \mathcal{K}^{*}$. Hence $\mathcal{K}^{*} \neq \mathrm{Z}_{1}, \mathrm{Z}_{1} \subset \mathcal{K}^{*}$. 
Note that one can show that if for a face $\mathcal{K}$, the set of zeros $T_{0}(\mathcal{K})$ consists of isolated elements then all these zeros are the minimal ones. In the next example, we will show that there exists an exposed face $\mathcal{K}(V, \mathcal{L})$ of $\mathcal{C O} \mathcal{P}^{p}$ with $V=\{\boldsymbol{\tau}(j), j \in I\}$ and $\mathcal{L}=\{L(j)=$ $\operatorname{supp}(\tau(j)), j \in I\}$, for which the set of zeros $T_{0}(\mathcal{K}(V, \mathcal{L}))$ consists of isolated elements $\tau(j), j \in J$, such that $I \subset J,|I|<|J|$, which illustrates that the number $|J|$ of the minimal zeros can be greater than the number $|I|$ of elements in the defining set $V$. To construct this face we will use some data from Example 5 in [19].

Example 5. Set $p=11$ and consider a $p \times p$ matrix $\mathbf{S}:=\left(\mathcal{P}^{p-1} \mathbf{a}, \ldots, \mathcal{P}^{1} \mathbf{a}\right.$, a $)$, where $\mathcal{P}=$ $\left(\mathbf{e}_{p}, \mathbf{e}_{1}, \ldots, \mathbf{e}_{p-1}\right), \mathbf{a}=(32,18,4,-24,-31,-31,-24,4,18,32,32)^{\top}$. It is shown in [19] that $\mathbf{S} \in \mathcal{C O} \mathcal{P}^{p}$ and it has 33 isolated zeros:

$$
\boldsymbol{\tau}(i)=\mathcal{P}^{i} \mathbf{u}, i=1, \ldots, 11 ; \boldsymbol{\tau}(i)=\mathcal{P}^{i} \mathbf{v}, i=12, \ldots, 22 ; \boldsymbol{\tau}(i)=\mathcal{P}^{i} \mathbf{w}, i=23, \ldots, 33,
$$

where $\mathbf{u}^{\top}=\frac{1}{21}(8,0,3,0,0,0,10,0,0,0,0), \mathbf{v}^{\top}=\frac{1}{21}(10,0,0,0,3,0,8,0,0,0,0)$, and $\mathbf{w}^{\top}=\frac{1}{7}(2,0,0,2,0,0,0,3,0,0,0)$.

Consider an exposed face

$$
\overline{\mathcal{K}}:=\left\{D \in \mathcal{C O} \mathcal{P}^{p}: D \bullet \mathbf{M}=0\right\}, \text { where } \mathbf{M}:=\sum_{i \in J} \boldsymbol{\tau}(i)(\boldsymbol{\tau}(i))^{\top}, J:=\{1, \ldots, 33\} .
$$

By construction,

$$
(\boldsymbol{\tau}(i))^{\top} D \boldsymbol{\tau}(i)=0 \forall i \in J, \forall D \in \overline{\mathcal{K}},
$$

$\mathbf{S} \in \overline{\mathcal{K}}, \mathbf{t}^{\top} \mathbf{S t}>0 \forall \mathbf{t} \in T \backslash\{\boldsymbol{\tau}(i), i \in J\}, \mathbf{e}_{k}^{\top} \mathbf{S} \boldsymbol{\tau}(i)>0 \forall k \in[p] \backslash \operatorname{supp}(\boldsymbol{\tau}(i)), \forall i \in J$.

It follows from the relations above that

$$
T_{0}(\overline{\mathcal{K}})=\{\boldsymbol{\tau}(j), j \in J\}=V_{0} \text { and } \mathcal{M}=\{M(j)=\operatorname{supp}(\boldsymbol{\tau}(j)), j \in J\}
$$

Hence, according to Lemma 3 , the face $\overline{\mathcal{K}}$ admits a representation $\overline{\mathcal{K}}=\mathcal{C O} \mathcal{P}^{p} \cap \mathcal{H}\left(V_{0}, \mathcal{M}\right)$, where subspace $\mathcal{H}\left(V_{0}, \mathcal{M}\right)$ is defined in (13).

One can check that $\mathcal{H}\left(V_{0}, \mathcal{M}\right)=\mathcal{H}(V, \mathcal{L})$, where $\mathcal{H}(V, \mathcal{L})$ is defined in (6), and

$$
V=\{\boldsymbol{\tau}(i), i \in I\}, \mathcal{L}=\{L(i)=\operatorname{supp}(\boldsymbol{\tau}(i)), i \in I\}, I=\{12, \ldots, 30\} \backslash\{27\},|I|=18
$$

Hence $\overline{\mathcal{K}}=\mathcal{C O} \mathcal{P}^{p} \cap \mathcal{H}(V, \mathcal{L})=: \mathcal{K}(V, \mathcal{L})$, where $|V|=18$ and the face $\overline{\mathcal{K}}$ has 33 minimal zeros $\tau(j), j \in J$.

Based on Theorem 1, we can easily determine the dimension of $\overline{\mathcal{K}}$ :

$$
\operatorname{dim} \overline{\mathcal{K}}=\operatorname{dim} \mathcal{H}\left(V_{0}, \mathcal{M}\right)=12
$$

\section{Summary of the Results and Conclusions}

The main contribution of the paper is to study the properties of faces of the copositive cone $\mathcal{C O} \mathcal{P}^{p}$. The concepts of zeros and minimal zeros were formulated for the case of an arbitrary face $\mathcal{K}$ of the cone of copositive matrices and allowed us to obtain various representations of this face, an explicit representation of the subspace generated by it, and to describe the minimal exposed face containing $\mathcal{K}$.

Summarizing the results of the paper, let us consider a few possible scenarios for their application, starting with the strongest and most effective one.

Given a face $\mathcal{K}$ of $\mathcal{C O}{ }^{p}$, the following sets (the data) are related to it:

1. the corresponding set $T_{0}(\mathcal{K})$ of all zeros of $\mathcal{K}$,

2. the set $V_{0}$ of minimal zeros of $\mathcal{K}$ (the set of vertices of the set $\operatorname{conv} T_{0}(\mathcal{K})$ ),

3. the set $\mathcal{M}$ defined in (11),

4. some sets $V$ and $\mathcal{L}$ such that the cone $\mathcal{K}$ admits representation (5). 
Suppose we do not have complete information about the cone $\mathcal{K}$, as only some of the sets from the list above are given. Let us see what information we can easily acquire in the case of incomplete data. The following scenarios are possible here.

A. In the case when the finite data sets enumerated in 2. and 3. are given, we can

(a1) explicitly describe the corresponding face of the cone $\mathcal{C O P}^{p}$ :

$$
\mathcal{K}=\mathcal{K}\left(V_{0}, \mathcal{M}\right)=\left\{D \in \mathcal{C O} \mathcal{P}^{p}: \mathbf{e}_{k}^{\top} D \boldsymbol{\tau}(j)=0, k \in M(j), j \in J\right\} ;
$$

(a2) easily construct the set $T_{0}(\mathcal{K})$ (see Proposition 3);

(a3) explicitly describe (without using the closure operator) the cone $\mathcal{K}^{*}$ that is dual to the face $\mathcal{K}$ (see (55));

(a4) explicitly describe the minimal exposed face $\mathcal{K}_{\text {exp }}^{\min }$ containing the face $\mathcal{K}$ and check whether the face $\mathcal{K}$ is exposed (see Theorems 2 and 3);

(a5) determine $\operatorname{span}(\mathcal{K})=\mathcal{H}\left(V_{0}, \mathcal{M}\right)$ and

$$
\operatorname{dim}(\mathcal{K})=\operatorname{dim}\left(\mathcal{H}\left(V_{0}, \mathcal{M}\right)\right) \leq \operatorname{dim}(\mathcal{H}(V, \mathcal{L})),
$$

where $\mathcal{H}\left(V_{0}, \mathcal{M}\right):=\left\{D \in \mathcal{S}^{p}: \mathbf{e}_{k}^{\top} D \tau(j)=0, k \in M(j), j \in J\right\}$.

B. Suppose that only the set $T_{0}(\mathcal{K})$ (the first set in the list 1.-4.) is given. In this case, we can only do the following:

(b1) find the set of vertices $V_{0}=\{\tau(j), j \in J\}$ of the set $\operatorname{conv} T_{0}(\mathcal{K})$;

(b2) construct the sets $M_{*}(j), j \in J$, defined in (37), and hence

(b3) explicitly describe the minimal exposed face $\mathcal{K}_{\text {exp }}^{\min }$ containing the face $\mathcal{K}$ but not the face $\mathcal{K}$ itself.

C. Suppose that the sets $V$ and $\mathcal{L}$ (see (2), (7)) defining the face are given, but it's unknown whether the pair $\{V, \mathcal{L}\}$ of these sets is the same as the pair $\left\{V_{0}, \mathcal{M}\right\}$ (i.e., it is possible that $\{V, \mathcal{L}\} \neq\left\{V_{0}, \mathcal{M}\right\}$ ). In this case the only guaranteed result consists of the explicit description of the face $\mathcal{K}$ by the rule (5).

D. Suppose that additionally to the data given in Scenario $C$ (i.e., the pair of the sets $\{V, \mathcal{L}\}$, defining the face $\mathcal{K}=\mathcal{K}(V, \mathcal{L})$ by the rule (5)), the set $V_{0}=\{\boldsymbol{\tau}(j), j \in J\}$ of minimal zeros of $\mathcal{K}$ is given. In this case one can easily find the sets $M(j), j \in J$, and hence provide all the constructions described in the items (a1)-(a5) of Scenario A.

Remark 5. From the above considerations, it follows that the data set $\left\{V_{0}, \mathcal{M}\right\}$ is the most useful finite data set for the corresponding face. In some cases, for $\mathcal{K}=\mathcal{K}(V, \mathcal{L})$, the set $V_{0}$ can be constructed based on the available data $V$ and $\mathcal{L}$. From Proposition 1 , it follows that having $V_{0}, V$ and $\mathcal{L}$, one can find the set $\mathcal{M}$, solving several Linear Programming problems.

In general case, the problem of finding the set $V_{0}$ is not trivial, but is very important and deserves a separate consideration.

The results of the paper may help to better understand the facial structure of the cone of copositive matrices and this knowledge can be effectively used in the duality theory of copositive optimization. The explicit descriptions of the faces of the copositive cone and their dual cones can be used in constructive regularization procedures based on the face reduction approach.

Author Contributions: Conceptualization, O.K.; methodology, O.K.; formal analysis, O.K. and T.T.; investigation, O.K. and T.T.; writing — original draft preparation, O.K. and T.T.; writing — review and editing, O.K. and T.T. All authors have read and agreed to the published version of the manuscript.

Funding: This research was partially supported by the state research program "Convergence" (Republic Belarus), Task 1.3.01, by Portuguese funds through CIDMA-Center for Research and Development in Mathematics and Applications, and FCT-Portuguese Foundation for Science and Technology, within the project UIDB/04106/2020. 
Institutional Review Board Statement: Not applicable.

Informed Consent Statement: Not applicable.

Data Availability Statement: Not applicable.

Acknowledgments: The authors thank the anonymous reviewers whose important comments and suggestions helped to significantly improve the presentation of this paper.

Conflicts of Interest: The authors declare no conflict of interest.

\author{
Abbreviations \\ LP Linear Programming \\ CoP Copositive Programming \\ SIP Semi-infimite Programming \\ SDP Semidefinite Programming
}

The following abbreviations are used in this manuscript:

\title{
References
}

1. Ahmed, F.; Dür, M.; Still, G. Copositive Programming via semi-infinite optimization. J. Optim. Theory Appl. 2013, 159, 322-340. [CrossRef]

2. Bomze, I.M. Copositive optimization-Recent developments and applications. EJOR 2012, 216, 509-520. [CrossRef]

3. Dür M. Copositive Programming-A survey. In Recent Advances in Optimization and Its Applications in Engineering; Diehl, M., Glineur, F., Jarlebring, E., Michielis, W., Eds.; Springer: Berlin/Heidelberg, Germany, 2010; pp. 3-20.

4. Anjos, M.F.; Lasserre, J.B. (Eds.) Handbook of Semidefinite, Conic and Polynomial Optimization; International Series in Operational Research and Management Science; Springer: Berlin, Germany, 2012; Volume 166, 138p.

5. Wolkowicz, H.; Saigal, R.; Vandenberghe, L. (Eds.) Handbook of Semidefinite Programming-Theory, Algorithms, and Applications; Kluwer Academic Publishers: Dordrecht, The Netherlands, 2000.

6. Drusvyatskiy, D.; Wolkowicz, H. The many faces of degeneracy in conic optimization. Found. Trends Optim. 2017, 3, 77-170. [CrossRef]

7. Pataki, G. The geometry of semidefinite programming. In Handbook of Semidefinite Programming; Wolkowicz, H., Saigal, R., Vandenberghe, L., Eds.; Springer: Boston, MA, USA, 2000; pp. 29-65.

8. Ramana, M.V. An exact duality theory for Semidefinite Programming and its complexity implications. Math. Prog. 1997, 77, 129-162. [CrossRef]

9. Ramana, M.V.; Tunçel, L.; Wolkowicz, H. Strong duality for Semidefinite Programming. SIAM J. Optim. 1997, 7, 641-662. [CrossRef]

10. Afonin, A.; Hildebrand, R.; Dickinson, P. The extreme rays of the $6 \times 6$ copositive cone. J. Glob. Optim. 2021, 79, 153-190. [CrossRef]

11. Dickinson, P.J.C. Geometry of the copositive and completely positive cones. J. Math. Anal. Appl. 2011, 380, 377-395. [CrossRef]

12. Hildebrand, R. Extremal copositive matrices with zero supports of cardinality $n-2$. Electron. J. Linear Algebra 2018, 34, 28-34. [CrossRef]

13. Berman, A.; Dür, M.; Shaked-Monderer, N. Open problems in the theory of completely positive and copositive matrices. Electron. J. Linear Algebra 2015, 29, 46-58. [CrossRef]

14. Pataki, G. On the connection of facially exposed and nice cones. J. Math. Anal. Appl. 2013, 400, 211-221. [CrossRef]

15. Kostyukova, O.I.; Tchemisova, T.V. On equivalent representations and properties of faces of the cone of copositive matrices. arXiv 2020, arXiv:2012.03610v1.

16. Kostyukova, O.I.; Tchemisova, T.V. Optimality conditions for convex Semi-Infinite Programming problems with finitely representable compact index sets. J. Optim. Theor Appl. 2017, 175, 76-103. [CrossRef]

17. Kostyukov, O.I.; Tchemisova, T.V.; Dudina, O.S. Immobile indices and CQ-free optimality criteria for linear copositive programming problems. Set-Valued Var. Anal. 2020, 28, 89-107. [CrossRef]

18. Boyd, S.; Vandenberghe, L. Convex Optimization; Cambridge University Press: Cambridge, UK, 2004.

19. Bomze, I.M.; Schachinger, W.R.; Ullrich, R. From seven to eleven: Completely positive matrices with high cp-rank. Linear Algebra Appl. 2014, 459, 208-221. [CrossRef] 